\title{
Comparison of PLIF and CFD Results for the Orion CEV RCS Jets
}

\author{
Christopher B. Ivey* \\ Stanford University, Stanford, CA 94305-3030 \\ Paul M. Danehy, ${ }^{\dagger}$ Brett F. Bathel, ${ }^{\ddagger}$ Artem A. Dyakonov, ${ }^{\S}$ Jennifer A. Inman, ${ }^{* *}$ and \\ Stephen B. Jones ${ }^{\dagger \dagger}$ \\ NASA Langley Research Center, Hampton, VA 23681-2199
}

\begin{abstract}
Nitric-oxide planar laser-induced fluorescence (NO PLIF) was used to visualize and measure centerline streamwise velocity of the Orion Crew Exploration Vehicle (CEV) Reaction Control System (RCS) Jets at NASA Langley Research Center's 31-Inch Mach 10 Air wind tunnel. Fluorescence flow visualizations of pitch, roll, and yaw RCS jets were obtained using different plenum pressures and wind tunnel operating stagnation pressures. For two yaw RCS jet test cases, the PLIF visualizations were compared to computational flow imaging (CFI) images based on Langley Aerothermal Upwind Relaxation Algorithm (LAURA) computational fluid dynamics (CFD) simulations of the flowfield. For the same test cases, the streamwise velocity measurements were compared to CFD. The CFD solution, while showing some unphysical artifacts, generally agree with the experimental measurements.
\end{abstract}

\section{Nomenclature}

$\begin{array}{ll}\text { AoA } & =\text { Angle of attack } \\ A_{21} & =\text { Einstein spontaneous emission coefficient }(1 / \mathrm{s}) \\ B_{12} & =\text { Einstein absorption coefficient }(1 / \mathrm{s}) \\ B_{v} & =\text { rotational constant for NO molecule }(1 / \mathrm{m}) \\ c & =\text { speed of light }(\mathrm{m} / \mathrm{s}) \\ f_{B} & =\text { Boltzmann population fraction } \\ g & =\text { spectral overlap integral } \\ g_{a} & =\text { spectral absorption profile } \\ g_{L} & =\text { spectral laser profile } \\ h & =\text { Planck constant }(\mathrm{J} \cdot \mathrm{s}) \\ i & =\text { fluorescence transition number } \\ J & =\text { rotational quantum number } \\ k & =\text { Boltzmann constant }(\mathrm{J} / \mathrm{K}) \\ m_{N O} & =\text { mass of NO }(\mathrm{kg}) \\ m_{N_{2}} & =\text { mass of } \mathrm{N}_{2}(\mathrm{~kg}) \\ m_{\mathrm{O}_{2}} & =\text { mass of } \mathrm{O}_{2}(\mathrm{~kg}) \\ N O & =\text { nitric oxide molecule } \\ N_{2} & =\text { nitrogen molecule }\end{array}$

\footnotetext{
* Graduate Student, Stanford University, Building 530, 440 Escondido Mall, AIAA Student Member.

${ }^{\dagger}$ Research Scientist, Advanced Sensing and Optical Measurement Branch, MS 493, AIAA Associate Fellow.

${ }^{\ddagger}$ Graduate Student, University of Virginia, and NASA Graduate Coop, AIAA Student Member.

§ Aerospace Engineer, Atmospheric Flight \& Entry Systems Branch, MS 489.

** Research Scientist, Advanced Sensing \& Optical Measurement Branch, MS 493, AIAA Member.

${ }^{\dagger+}$ Research Technician, Advanced Sensing and Optical Measurement Branch, MS 493.
} 


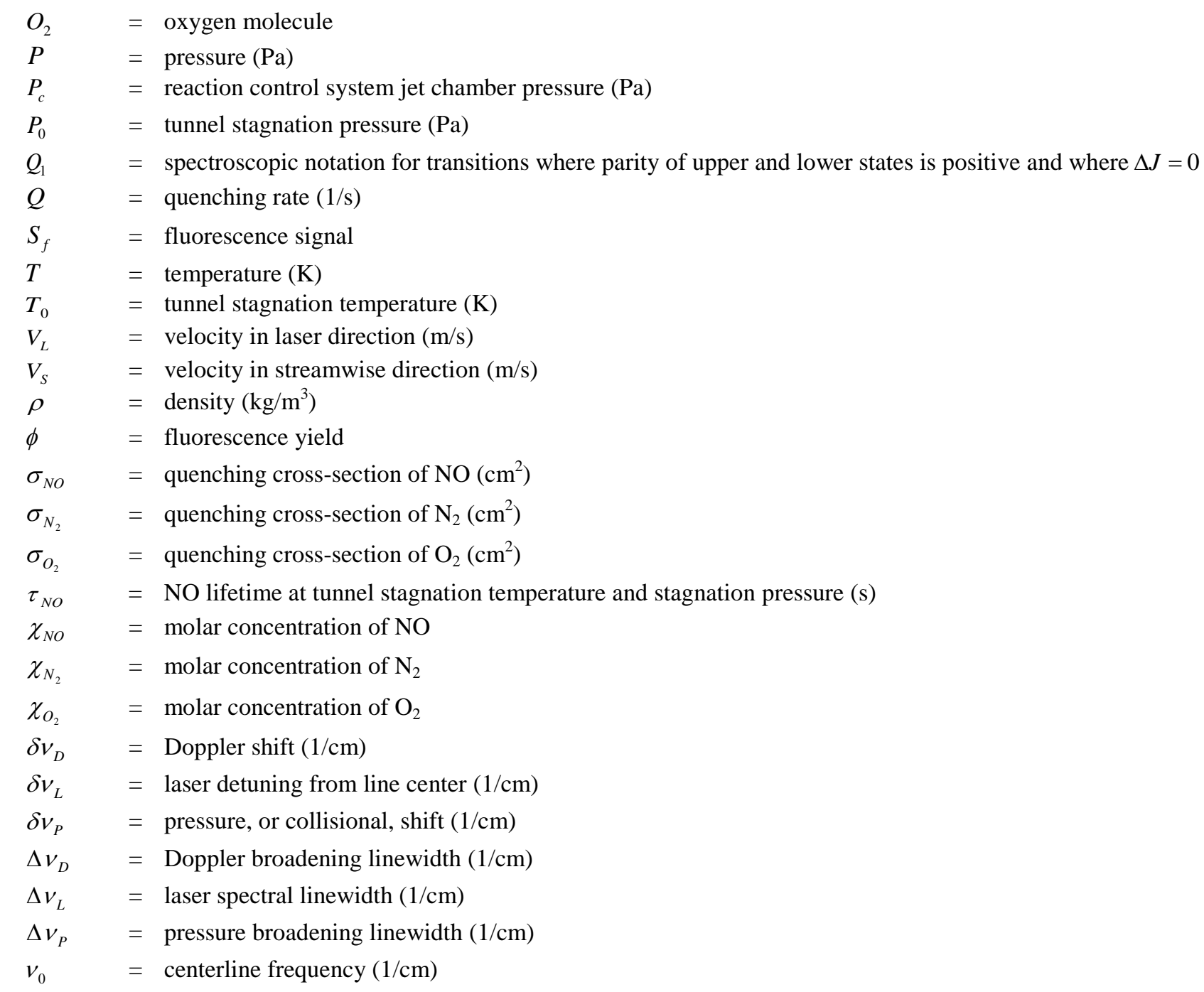

\section{Introduction}

COMPARATIVE experimental studies are necessary for the qualification and development of accurate

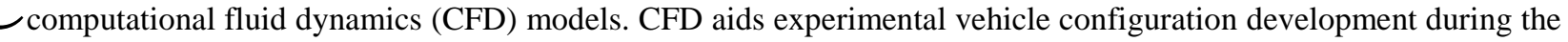
iterative design process by lowering overall costs and reducing the turn-around time on designs. On the other hand, CFD models need to be validated against experimental data over a wide range of conditions to justify assumptions and verify model constants. This need is amplified when the simulations involve complicated physical processes that are typical of flows associated with hypersonic flight vehicles. The forebody flow of such vehicles include the effects of ablation, real gas effects, and thermal and chemical nonequillibrium, while the wake flow include flow separation, shear layers, and plumes. The transition to turbulence in both forebody and wake flows of these vehicles further complicates CFD modeling. In this paper, we consider such a wake flow: the aftbody flowfield of the Orion Crew Exploration Vehicle (CEV) upon reentry with the use of Reaction Control System (RCS) jets.

In ground test facilities used to simulate reentry, few suitable spatially and temporally resolved flowfield measurement techniques exist. In this study, nitric oxide planar laser-induced fluorescence (NO PLIF) imaging and molecular-tagging velocimetry (MTV) are used to obtain three-dimensional visualizations and centerline streamwise velocities of the RCS Jets, respectively. The intensity of any given pixel in a PLIF image depends on many local flow parameters in addition to information about the optical system setup, complicating the extraction of any single local property. By modeling the fluorescence signal, direct comparisons between CFD predictions and the experiment can be made. This method is known as computational flow imaging (CFI) and has been applied to study similar hypersonic flows. ${ }^{1-4}$ In this study, qualitative comparisons are made between CFI and experimental fluorescence images of the RCS jets interacting with the wake flow of the Orion CEV. The MTV velocity measurements with known uncertainties provide a quantitative means to compare the CFD and PLIF centerline streamwise velocities. The purpose of this paper 
is both to report PLIF visualizations and velocity measurements of the Orion CEV RCS Jets and to evaluate a more detailed approach for comparing PLIF and CFD results.

\section{A. Test Facility}

\section{Experiment and Procedures}

The experiments were performed in the 31-Inch Mach 10 Air Tunnel facility at NASA Langley Research Center. The facility has a nominal Mach number of 9.7 with a nominal stagnation temperature, $T_{0}$, of 1,000 K. Three large UVgrade fused silica windows, transparent in the ultraviolet down to approximately $190 \mathrm{~nm}$, form three walls (top, side and bottom) of the 31-inch square test section and a model injection system forms the fourth side wall. The top window provides access for the laser sheet to interrogate the flow, and the side window provides access to image the interrogated flow. Two facility stagnation pressures, $P_{0}$, were investigated: $350 \mathrm{psi}$ (2.41 MPa) and 1,300 psi (9.30 $\mathrm{MPa}$ ). Further details of the flow properties at these conditions can be found in Hollis et al. ${ }^{5}$ or from the authors. Run durations lasted for approximately 1 minute. For a thorough description of the facility, please see the paper by Micol. ${ }^{6}$

\section{B. Test Model}

The experiments were conducted on a 0.025 scale (5-inch diameter) Orion Crew Exploration Vehicle (CEV) model, shown in Fig. 1. It had inserts containing 10-degree half angle conical Reaction Control System (RCS) jets with $0.7 \mathrm{~mm}$ (0.0275 in.) throat diameter and with an area ratio of 13.4. The RCS consisted of yaw, pitch and roll nozzle jet pairs. In the present paper, only data from single-nozzles are presented. Jet pairs were also studied during the wind tunnel tests. The jet fluid composition was maintained at approximately $4 \%$ $\mathrm{NO}$ and $96 \% \mathrm{~N}_{2}$. Three model jet chamber pressures, $P_{\mathrm{c}}$, were investigated: $\sim 45$ psi $(0.31$ $\mathrm{MPa}), \sim 132 \mathrm{psi}(0.91 \mathrm{MPa})$, and $\sim 425 \mathrm{psi}$ (2.93 $\mathrm{MPa})$. The axis of symmetry of the model was oriented at an $18^{\circ}$ AoA with respect to the free stream. Reference 7 contains more information about the model.

\section{Laser System}

The laser system consisted of three main components: a pump laser, a dye laser, and a frequency-mixing unit. The second and third harmonics of a pulsed Spectra Physics Pro-230

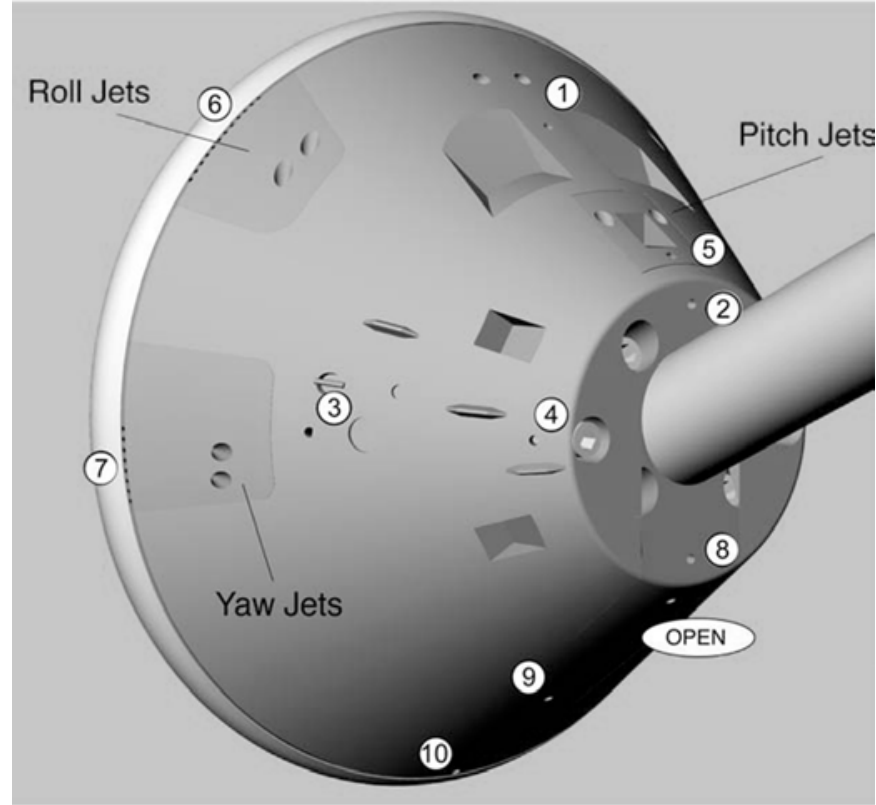

Figure 1: Orion Command Module RCS Test Model (606DTSP) assembled view with numbered pressure ports and jet locations (from Reference 7).

Nd:YAG laser, at wavelengths of $532 \mathrm{~nm}$ and $355 \mathrm{~nm}$ respectively, comprised the pump laser. The $532 \mathrm{~nm}$ output was used to pump a Sirah Cobra Dye laser to achieve a $622 \mathrm{~nm}$ output. The $622 \mathrm{~nm}$ output of the dye laser was then mixed with the $355 \mathrm{~nm}$ output of the pump laser in a Sirah Frequency Conversion Unit to produce $\sim 5 \mathrm{~mJ}$ per $10 \mathrm{~ns}$ pulse at $226.256 \mathrm{~nm}$. This wavelength was chosen to excite strongly fluorescing spectral lines of NO near the $Q_{1}$ band head. In this notation, $Q$ denotes that the rotational quantum number, $J$, is the same for both the upper and lower states of the transition, i.e, $\Delta J=0$. The subscript 1 indicates that the parity of both the upper and lower states involved in the transition is positive, meaning that in both states, the electronic spin is aligned with the total angular momentum of the molecule.

To form the laser sheet, the collimated $226.256 \mathrm{~nm}$ output was passed through a $36 \mathrm{~mm}$ focal length cylindrical lens, oriented to diverge the beam in the direction parallel to the free stream. It was then passed through a $1 \mathrm{~m}$ focal length spherical lens that collimated it in the freestream direction and focused it in the spanwise direction, forming a 75 mm wide by $\sim 0.5 \mathrm{~mm}$ thick sheet in the test section. For experiments involving molecular tagging velocimetry (MTV), multiple lines were required to tag NO. Therefore, a filter (a fine-toothed hair comb) was placed between the optics and the test section to form $\sim 1 \mathrm{~mm}$ line widths (FWHM) with $\sim 2 \mathrm{~mm}$ gaps. Further details of the laser system can be found in Bathel et al. ${ }^{8}$ 


\section{Camera System}

For the flow visualization experiments, a Princeton Instruments PI-MAX II with a gated image intensifier and a $512 x 512$ pixel CCD array was used. Custom filters (with $<1 \%$ transmission at $226 \mathrm{~nm}$ and $>80 \%$ transmission at 235$280 \mathrm{~nm}$, made by Layertec $\mathrm{GmbH}$ ) were used to transmit the fluorescence signal while rejecting the laser scatter. Images were acquired using a $45 \mathrm{~mm}$ F/1.8 UV lens (Cerco Model type 2073, manufactured by EADS Sodern), achieving a spatial resolution of about 2.7 pixels $/ \mathrm{mm}$. NO fluorescence was imaged at a viewing angle normal to the laser sheet at a rate of $10 \mathrm{~Hz}$, matching the laser pulse rate, with flow-stopping time resolution ( $1 \mu \mathrm{s})$.

For the MTV experiments, a Cooke Dicam Pro with an intensified 1280x1024 pixel array interline progressive scan CCD was used. Only a 1280x288 pixel region was used so that an image read-out rate of $10 \mathrm{~Hz}$, corresponding to the laser pulse frequency, could be achieved. A Nikon $105 \mathrm{~mm}$ F/4.5 UV-Nikkor lens was used, achieving a spatial resolution of 17.5 pixels $/ \mathrm{mm}$. The camera was operated in double shutter mode, wherein the camera was capable of acquiring an image pair with a $500 \mathrm{~ns}$ temporal separation (between the end of the first gate and the beginning of the second). The first intensifier gate duration was $20 \mathrm{~ns}$, the minimum duration possible for this camera. Once a gas molecule has been tagged (excited by the laser), the fluorescence intensity decays exponentially in time, where the exponential time constant depends on the local pressure, temperature and gas composition. The Cooke Dicam Pro does not allow for independent gain settings for the first and second exposure. Therefore, in order to have a measurable second exposure, it was necessary to use the minimum gate delay (500 ns) and to keep the second gate open long enough (300 ns) to collect a sufficient number of photons. Furthermore, it was necessary to attenuate the first exposure by using the minimum gate duration setting (20 ns) and by advancing the camera gate $15 \mathrm{~ns}$ with respect to the laser excitation to create a shorter effective gate of 5 to $10 \mathrm{~ns}$. These settings resulted in comparable intensities between the first and second exposures. The camera and the laser are triggered by a LabSmith LC880 Programmable Logic Controller to create a relative delay between the laser excitation and first exposure. Fine adjustments to the first gate duration were made by varying co-axial cable length between the trigger and the camera, yielding delay adjustment increments on the order of 1 ns (whereas the camera software allows delay adjustments only in coarse increments of 20 ns). In summary, the effective duration of the first gate was between 5-10 ns, the delay between the end of the first gate and the beginning of the second was $500 \mathrm{~ns}$, and the duration of the second gate was $300 \mathrm{~ns}$. For a thorough description of the velocity measurement technique and more details about the camera setup, please see Bathel et al. ${ }^{8}$

\section{E. PLIF Flow Visualization}

The $\sim 75 \mathrm{~mm}$ wide laser sheet is directed into the top tunnel window with the sheet oriented parallel to the freestream and normal to the camera system, which views the model and flow from the side window. The laser sheet excites the seeded NO molecules in the jet fluid. The resulting fluorescence is then imaged by the camera system. The laser sheet was scanned spatially through the flow using optics attached to a translation stage to visualize different cross-sections of the jet fluid. During such scans, the camera is fixed, so the magnification of the images varies by several percent. To account for the change in magnification, as well as lens and perspective distortion, a series of reference images were taken of a dotcard, which is a sheet marked with equally spaced square dots at a known position. ${ }^{9}$ Each run investigated a single actuated RCS jet operated at two jet chamber pressures and a constant tunnel stagnation pressure. The test sequence is shown in Table 1. Further details on the PLIF flow visualization experimental procedure can be found in the paper by Danehy et al. ${ }^{10}$

Table 1. Test Matrix. Two conditions (denoted by an A or B appended to the Run number) were tested in each run.

\begin{tabular}{ccccc}
\hline \hline Run $\#$ & Run Type & RCS Jet & $\mathbf{P}_{\mathbf{0}} \mathbf{( p s i )}$ & $\mathbf{P}_{\mathbf{c}} \mathbf{( \mathbf { p s i } )}$ \\
\hline 4A & PLIF Visualization & Pitch & 350 & 45 \\
4B & PLIF Visualization & Pitch & 350 & 136 \\
3A & PLIF Visualization & Pitch & 1300 & 145 \\
3B & PLIF Visualization & Pitch & 1300 & 425 \\
5A & PLIF Visualization & Roll & 350 & 44 \\
5B & PLIF Visualization & Roll & 350 & 130 \\
6A & PLIF Visualization & Roll & 1300 & 144 \\
6B & PLIF Visualization & Roll & 1300 & 420 \\
10A & PLIF Visualization & Yaw & 350 & 45 \\
10B & PLIF Visualization & Yaw & 350 & 132 \\
11A & PLIF Visualization & Yaw & 1300 & 144 \\
11B & PLIF Visualization & Yaw & 1300 & 424 \\
17A & PLIF MTV & Yaw & 350 & 44 \\
17B & PLIF MTV & Yaw & 350 & 131 \\
\hline \hline
\end{tabular}




\section{F. PLIF Molecular Tagging Velocimetry}

In order to perform velocimetry, a slight modification is made to the laser sheet used for flow visualization: after optics form the beam into a sheet, a fine-toothed hair comb is placed between the optics and the test section to form 1 $\mathrm{mm}$ line widths with $\sim 2 \mathrm{~mm}$ gaps, as described above in Laser System. The laser comb is directed vertically downward with the $\sim 75 \mathrm{~mm}$ side being parallel to free stream and normal to the camera system, as the laser sheet is in PLIF flow visualization. For the velocimetry data reported herein, the laser comb was not translated spatially but rather remained fixed at the jet center so repeated frames could be acquired. As in flow visualization, the laser excites the seeded NO molecules in the jet fluid. The resulting fluorescence is sequentially imaged twice by the camera system, where the first acquisition occurs at excitation and the second acquisition occurs after the excited lines of NO convect downstream for $500 \mathrm{~ns}$, as described above in Camera System. The single velocimetry run reported herein acquired multiple image pairs at the jet centerline. This run investigated the yaw RCS jet while operating at two jet chamber pressures and a single tunnel stagnation pressure. To account for lens and perspective distortions, reference dotcard images were again taken at several locations in the flowfield. For details of the run parameters, please see Table 1. Further details on the PLIF MTV experimental procedure can be found in the paper by Bathel et al. ${ }^{8}$

\section{Data Analysis and Computational Methodology}

\section{A. Flow Visualization Image Processing and Analysis}

Images are first processed to remove distortions associated with the imaging system. The UnwarpJ function of the National Institute of Health's ImageJ program is used to apply this correction. An image acquired of the dotcard located at the model centerline is spatially transformed using UnwarpJ so that the dots are equally spaced and are aligned in vertical and horizontal rows to match the physical dotcard. The transformation is then applied to the dotcard images taken away from the model centerline and to the PLIF images to remove lens and perspective distortion. The background fluorescence is then subtracted from the PLIF images and a false color map is applied. In the Virtual Diagnostics Interface (ViDI), the transformed dotcard images are positioned relative to the Orion model according to their known offsets from the jet centerline. The dotcard images taken away the model centerline are scaled to match the dot size, placement and spacing of the actual physical dotcard. Linear functions are fit to the position and scaling information in such a way that the image offset from the jet centerline dictates relative position and scale. This procedure corrects for the variation in magnification caused by scanning the laser sheet position. Subsequently, PLIF images are imported into ViDI and aligned with the model with accuracy slightly less than $1 \mathrm{~mm}$. The PLIF visualizations are positioned and scaled according to their recorded offsets and the linear functions obtained from the dotcard images, resulting in a three-dimensional representation of the jet. Further details on using ViDI to display PLIF images can be found in the paper by Inman et al. ${ }^{11}$

\section{B. Molecular Tagging Velocimetry Image Processing and Analysis}

In brief, images acquired during the second camera gate are compared to the corresponding images acquired during the first camera gate. The displacement of the fluorescing lines of NO as a function of the relative time delay between the images is used to determine the flow velocity perpendicular to the lines. A 1-dimensional cross-correlation method is used to determine the physical shift of the lines. For a thorough description of the MTV processing and corresponding error analysis methods used for the Orion CEV RCS Jets, please see Bathel et al. ${ }^{8}$

\section{Computational Fluid Dynamics}

The CFD simulations shown in this paper were performed for a range of wind tunnel conditions and RCS nozzle flow conditions using Langley Aerothermal Upwind Relaxation Algorithm (LAURA) code. ${ }^{12,13}$ Calculations used a laminar flow model with air as a perfect gas both in the free stream and in the nozzles. Nozzle grids extended to the throat, allowing some capture of the internal flow gradients. The nominal tunnel free stream conditions were used. The nozzle throat conditions were calculated assuming a 1-D isentropic air nozzle flow. Calculations used a half-symmetric domain to save computational resources. This was thought adequate in the present case of the yaw nozzles, which are a sufficient distance from the centerline that the results should not be affected by the introduction of the artificial symmetry boundary. Grids contained from $6.5 \mathrm{M}$ to $52 \mathrm{M}$ points, though the solution used here was computed on a 13Mpoint grid. The standard LAURA bow shock and boundary layer adaptation was used, and the capsule's wake grid was kept as short as is practical to keep up the grid density. Calculations used both local and global (time consistent) time integration with no noticeable difference in near-plume phenomena.

For velocity comparisons, streamwise velocity was extracted from the CFD after a coordinate transformation. For the CFD computations shown in this paper, both the wind tunnel fluid and the RCS jet fluid were assumed to be air, which was treated as a perfect gas. For the NO PLIF CFI calculations shown in this paper, the entire fluid domain was 
assumed to be composed of the experimental jet fluid $\left(\chi_{\mathrm{O}_{2}}=0, \chi_{\mathrm{N}_{2}}=0.96, \chi_{\mathrm{NO}}=0.04\right)$. Ideally, the wind tunnel fluid and the RCS jet fluid could be modeled as two distinct gases whose compositions could be varied independently. However, the CFD computations were performed with a single fluid, air, for both the wind tunnel and RCS jet fluids. CFI computations required that the composition contain some NO in order to perform calculations of fluorescence intensity (i.e., the fluid could not be assumed to be air, as in the CFD computations); this suggested two possible prescriptions for the composition: 1) air with some seeded NO, or 2) the experimental jet fluid composition of $\mathrm{N}_{2}$ seeded with NO. Prescription 1 was tested; however, the results showed significant, unphysical amounts of intensity decay near the nozzle exit (a high-pressure area) due to $\mathrm{O}_{2}$ quenching. Since the experimental jet fluid did not contain $\mathrm{O}_{2}$ (and since $\mathrm{NO}$ and $\mathrm{O}_{2}$ can react at high-pressures), a significant level of quenching only occurs in regions where the jet fluid has mixed with the wind tunnel fluid, which contains $\mathrm{O}_{2}$, and not in the core of the jet plume. Thus, prescription 1 was a poor approximation of the experiment. Prescription 2 was also tested, and although it fails to account for $\mathrm{O}_{2}$ quenching in these mixing regions far from the jet centerline, the resulting jet-plume structures more closely matched the experimental visualizations; results presented in this paper therefore used this second prescription. The assumption of a single composition ( $\mathrm{N}_{2}$ seeded with $\mathrm{NO}$ ) as both the freestream and RCS jet gas did complicate the CFI processing, as described in the following section.

\section{Computational Flow Imaging}

The intensity at any given pixel of a PLIF image has a functional dependence on the local temperature, pressure, velocity, density, and species, as well as the laser setup. Equation (1) gives a simplified expression for the fluorescence intensity's dependence on the local state variables, where $S_{f}$ is the fluorescence intensity, $\phi$ is the fluorescence yield, $\chi_{N O}$ is the local mole fraction of NO, $\rho$ is the local gas density, $f_{B}$ is the Boltzmann fraction, $B_{12}$ is the Einstein absorption coefficient, $g$ is the spectral overlap integral, and where the summation occurs over all probed transitions, $i$. There are approximately six physically significant transitions of NO near $226.256 \mathrm{~nm}$, namely the strongly fluorescing spectral lines near the $Q_{1}$ band head. For the calculations herein, these are the only transitions that are investigated. Equation (1) is relevant to flows where NO is the probed gas and where there is negligible saturation of the molecular transitions and rapid repopulation of the laser-excited ground state. ${ }^{1,3,4}$

$$
S_{f} \propto \phi \cdot \chi_{N O} \cdot \rho \cdot \sum_{i}\left[f_{B} \cdot B_{12} \cdot g\right]_{i}
$$

Equation (1) does not directly show how the fluorescence intensity depends on the local state parameters such as temperature and pressure since several of the terms are themselves functions of local state parameters, so the variables used within the relation will be further described herein. The quantity $\phi$ is simply the ratio of the number of photons emitted as fluorescence to the number of photons absorbed, given by Eq. (2), where $A_{21}$ is the Einstein spontaneous emission coefficient and $Q$ is the quenching rate. The quantity $A_{21}$ is given by $1 / \tau_{N O}$, where $\tau_{N O}$ is the NO lifetime at the tunnel stagnation temperature and pressure.

$$
\phi=\frac{A_{21}}{A_{21}+Q}
$$

The quantity $Q$ in Eq. (2) is the sum of the quenching rates due to all molecular species present, as shown in Eq. (3), where $k$ is the Boltzmann constant, $m_{N O}$ is the molecular mass of NO, $P$ is the local gas pressure, $T$ is the local gas temperature, $\sigma_{\mathrm{NO}}$ is the $\mathrm{NO}$ quenching cross-section, $\chi_{\mathrm{O}_{2}}$ is the local mole fraction of $\mathrm{O}_{2}, m_{\mathrm{O}_{2}}$ is the molecular mass of $\mathrm{O}_{2}, \sigma_{\mathrm{O}_{2}}$ is the $\mathrm{O}_{2}$ quenching cross-section, $\chi_{N_{2}}$ is the local mole fraction of $\mathrm{N}_{2}, m_{N_{2}}$ is the molecular mass of $\mathrm{N}_{2}$, and $\sigma_{N_{2}}$ is the quenching cross-section of $\mathrm{N}_{2}{ }^{3}$

$$
Q=\sqrt{\frac{8 \cdot k \cdot T}{\pi \cdot m_{\mathrm{NO}}}} \cdot \frac{P}{k \cdot T} \cdot\left[\chi_{\mathrm{NO}} \cdot \sqrt{1+\frac{m_{\mathrm{NO}}}{m_{\mathrm{NO}}}} \cdot \sigma_{\mathrm{NO}}+\chi_{\mathrm{O}_{2}} \cdot \sqrt{1+\frac{m_{\mathrm{NO}}}{m_{\mathrm{O}_{2}}}} \cdot \sigma_{\mathrm{O}_{2}}+\chi_{\mathrm{N}_{2}} \cdot \sqrt{1+\frac{m_{\mathrm{NO}}}{m_{\mathrm{N}_{2}}}} \cdot \sigma_{\mathrm{N}_{2}}\right]
$$

The quenching cross-sections are temperature dependent; however, a theoretical representation of the dependency is unavailable, necessitating the usage of empirical models. The quenching cross-section models used within this study, shown in Eq. (4), were taken from Settersten et al. ${ }^{14}$

$$
\begin{aligned}
\sigma_{\mathrm{NO}} & =\left[34.8 \cdot e^{48.5 / T}+96.0 \cdot e^{-2690 / T}\right] \cdot 10^{-20} \\
\sigma_{\mathrm{O}_{2}} & =\left[22.5 \cdot e^{61.9 / T}+7.41 \cdot e^{-3800 / T}\right] \cdot 10^{-20} \\
\sigma_{\mathrm{N}_{2}} & =\left[0.008+2.04 \cdot e^{-2250 / T}+96.0 \cdot e^{-12700 / T}\right] \cdot 10^{-20}
\end{aligned}
$$

The quantity $f_{B}$ of Eq. (1) is given by Eq. (5), where $h$ is the Plank constant, $c$ is the speed light, $B_{v}$ is the effective rotational constant for the NO molecule which includes the effects of vibration/rotation interaction, and $J$ is the rotational quantum number of the transition; development of this representation is found within the text by Eckbreth. ${ }^{15}$ 


$$
f_{B}=\frac{h \cdot c \cdot B_{v}}{k \cdot T}[2 \cdot J+1] e^{-B_{v} \cdot \frac{h \cdot c \cdot[J+1]}{k \cdot T}}
$$

Equation (6) defines the quantity $g$, where $g_{L}$ is the spectral laser profile (Lorentzian or Gaussian profile, depending on laser), $g_{a}$ is the spectral absorption profile (Voigt profile), and $v$ is the frequency. The Voigt profiles is a convolution of the Lorentzian-shaped pressure broadening profile with the Gaussian-shaped Maxwellian velocity distribution, which is associated with Doppler broadening. The spectral profile representations in Eq. (6) are developed in the text by Eckbreth. ${ }^{15}$ Evaluation of Eq. (6) requires numeric integration and/or a lookup table.

$$
\begin{aligned}
g & =\int_{-\infty}^{\infty} g_{L} \cdot g_{a} d v \\
g_{L, \text { Gaussian }}(v) & =\sqrt{\frac{4 \cdot \ln (2)}{\Delta v_{L}^{2} \cdot \pi} \cdot e^{-4 \cdot \ln (2) \cdot \frac{\left(v-v_{L}\right)^{2}}{\Delta v_{L}^{2}}}} \\
g_{L, \text { Lorentzian }}(v) & =\frac{1}{2 \cdot \pi} \cdot \frac{\Delta v_{L}}{\left(v-v_{L}\right)^{2}+\left[\frac{\Delta v_{L}}{2}\right]^{2}} \\
g_{a}(v) & =\frac{2 \cdot \ln (2) \cdot \Delta v_{P}}{\pi^{1.5} \cdot \Delta v_{D}^{2}} \cdot \int_{-\infty}^{\infty} \frac{e^{-y^{2}}}{\ln (2) \cdot\left[\frac{\Delta v_{P}}{\Delta v_{D}}\right]^{2}+\left[\frac{2 \cdot \sqrt{\ln (2)} \cdot\left(v-\left\langle v_{0}-\delta v_{D}-\delta v_{P}\right\rangle\right)}{\Delta v_{D}}-y\right]^{2}} d y
\end{aligned}
$$

For all representations, $\Delta v_{D}$ is the Doppler broadening width, $\Delta v_{P}$ is the pressure broadening width, $v_{L}$ is the center laser frequency, $\Delta v_{L}$ is the laser linewidth, $v_{0}$ is the center absorption frequency, $\delta v_{L}=v_{L}-v_{0}$ is the laser detuning from linecenter, $\delta v_{P}$ is the pressure shift and $\delta v_{D}$ is the Doppler shift.

To reduce computation time for volumetric calculations, the quantity $g_{a}$ was approximated by assuming that the pressure broadening, usually assumed to have a Lorentzian profile was instead a Gaussian profile of the same width. This double-Gaussian simplification to the Voigt profile has an analytic solution. Furthermore, if the quantity $g_{L}$ was represented by a Gaussian profile as well (the so-called triple-Gaussian assumption), the additional convolution is analytically solved. The resulting analytic representation of the quantity $g$, shown in Eq. (7). ${ }^{3}$

$$
g\left(v_{L}\right)=\sqrt{\frac{4 \cdot \ln (2)}{\pi \cdot\left[\Delta v_{P}^{2}+\Delta v_{D}^{2}+\Delta v_{L}^{2}\right]}} \cdot e^{-4 \cdot \ln (2) \cdot \frac{\left[\delta v_{P}+\delta v_{D}+\delta v_{L}\right]^{2}}{\Delta v_{P}^{2}+\Delta v_{D}^{2}+\Delta v_{L}^{2}}}
$$

Expressions for $\Delta v_{D}$ and $\delta v_{D}$ are known exactly and are given by Eq. (8), where $V_{L}$ is the gas velocity parallel to the laser beam's direction of propagation. ${ }^{15}$

$$
\begin{aligned}
& \Delta v_{D}=\frac{2 \cdot v_{0}}{c} \cdot \sqrt{\frac{2 \cdot \ln (2) \cdot k \cdot T}{m_{N O}}} \\
& \delta v_{D}=\frac{v_{0} \cdot V_{L}}{c}
\end{aligned}
$$

The quantities $\Delta v_{L}$, and $\delta v_{L}$ are determined from the experimental setup, whereas the quantities $\Delta v_{P}$ and $\delta v_{P}$ are modeled by Eq. (9), where the pressure $P$ is in $\mathrm{Pa}^{16}$

$$
\begin{gathered}
\Delta v_{P}=\frac{P}{101,325} \cdot\left[0.585 \cdot \chi_{N_{2}} \cdot\left[\frac{295}{T}\right]^{0.75}+0.527 \cdot \chi_{O_{2}} \cdot\left[\frac{295}{T}\right]^{0.66}+0.551 \cdot \chi_{N O}\right] \\
\delta v_{P}=\frac{P}{101,325} \cdot\left[-0.18 \cdot \chi_{N_{2}} \cdot \sqrt{\frac{295}{T}}-0.159 \cdot \chi_{O_{2}} \cdot\left[\frac{295}{T}\right]^{0.52}-0.167 \cdot \chi_{N O}\right]
\end{gathered}
$$

The validity of triple-Gaussian spectral profile is explored in Experimental Results and Comparison with CFD, where the CFI fluorescence signal at the jet centerline is presented for the Gaussian-Voigt spectral overlap integral, the 
spectral representation using the Gaussian laser profile and the Voigt absorption profile, at the highest jet pressure. Also compared in Experimental Results and Comparison with CFD is the simplified CFI expression shown in Eq. (10). This expression is a two dimensional polynomial fit for $T$ and $P$ and includes several assumptions. The assumptions include a rapid repopulation of the ground state, negligible saturation of the fluorescence transitions, a triple-Gaussian spectral overlap integral representation (assuming $\chi_{O_{2}}=0, \chi_{N_{2}}=0.995, \chi_{N O}=0.005, v_{L}=v_{0}$, and $\delta v_{D}=0$ for broadening and shift computations as well as quenching computations), a temperature range between $100 \mathrm{~K}$ and $500 \mathrm{~K}$, a pressure range between $2 \mathrm{~Pa}$ and 30,000 $\mathrm{Pa}$, and that $S_{f}$ is linear with $\chi_{N O}{ }^{4}$ A notable assumption is that quenching from $\mathrm{O}_{2}$ was neglected. At the time of formulation of Eq. (10), the updated quenching cross-sections of Eq. (4) were unavailable, so the quenching cross-sections from Paul et al. ${ }^{17}$ were used. Further information on the simplified CFI representation can be found in Wilkes et al. ${ }^{4}$

$$
S_{f, \text { Wilkes }} \propto \chi_{N O} \cdot\left[-0.0018 \cdot T^{-2.12} \cdot P^{2}+46.0 \cdot T^{-1.9} \cdot P\right]
$$

Please note that the remainder of this discussion will be focused on the CFI representation presented in Eq. (1), assuming the simplified $g$ presented in Eq. (7).

The CFD procedures assumed that both the jet and the ambient were a single fluid composition, which was air. In order to adapt the CFD solution to an acceptable form for NO PLIF CFI, all calculations assumed that the entire computational domain consisted of the exiting jet fluid ( $\chi_{\mathrm{O}_{2}}=0, \chi_{N_{2}}=0.96, \chi_{N O}=0.04$ ). This assumption ignores $\mathrm{O}_{2}$ quenching and results in a uniform computed background fluorescence outside the jet.

All CFI calculations were done in Tecplot $360^{\mathrm{TM}}$ CFD post-processing and visualization software. In order to reconstruct a three-dimensional CFI jet, the computational domain was sliced into a series of vertical planes, oriented parallel to the tunnel streamwise direction, like the laser sheet. The planes were taken to extend past the jet plume in the spanwise direction to approximate the full jet plume structure with a distinct number of planes, a procedure akin to scanning the laser sheet through the jet plume for NO PLIF. The CFD results are used to determine the computational fluorescence signal for each of the sliced planes. Each plane then has the background fluorescence (typically just a few $\%$ of the maximum signal) subtracted off to account for the false assumption that there was also NO in the ambient wind tunnel fluid. While this subtraction does affect the ratios of fluorescence intensities elsewhere in the images, it did not significantly change fluorescence intensities in the jet fluid. The planes are then cropped to only show the jet plume region. This procedure was used to remove high levels of artificial fluorescence from the bow shock at the forebody of the Orion CEV that resulted from assuming NO was everywhere within the domain. The planes then have the same false color maps applied as the experimental PLIF images. Because the computed fluorescence intensity is numerically proportional to the actual intensity recorded by the intensified CCD, the CFI images are scaled so that the pixel intensity at the jet exit of the CFI centerline image matches that of the PLIF centerline image. The CFI images are then imported into the ViDI environment, where the planes are placed with respect to the jet centerline plane based on their coordinates obtained from Tecplot $360^{\mathrm{TM}}$

\section{Flow Field Structure}

Figure 2 shows several of the flow features of the Orion CEV flow field, which is typical of hypersonic capsule-type blunt body flows. Hypersonic blunt body flows are characterized by presence of a bow shock, which decelerates and compresses the oncoming flow. After passing through the shock wave, the gas expands outward in the radial direction towards the edge of the capsule's heatshield, causing the local Mach number to increase. The pressure difference between the forebody and aftbody drives the flow to expand supersonically around the model's shoulder (junction between the heat shield and the aftbody). Under some conditions the flow will separate and form a supersonic mixing layer, or shear layer, a boundary between the external flow and lower-speed recirculating flow. For example, on the leeward side of the model, the flow separates on the aftbody, as shown in Fig. 2. Finally the outer flow is processed by a recompression shock wave, turning the gas downstream. The size of the recirculation zone is sensitive to free-stream properties, model shape, vehicle AoA, gas chemistry, levels of turbulence and surface roughness, to name several (but not all) import factors. Flow on the windward side of the model can stay attached to the model surface, as shown in Fig. 2. This gas is also processed by a recompression shock downstream of the capsule. 


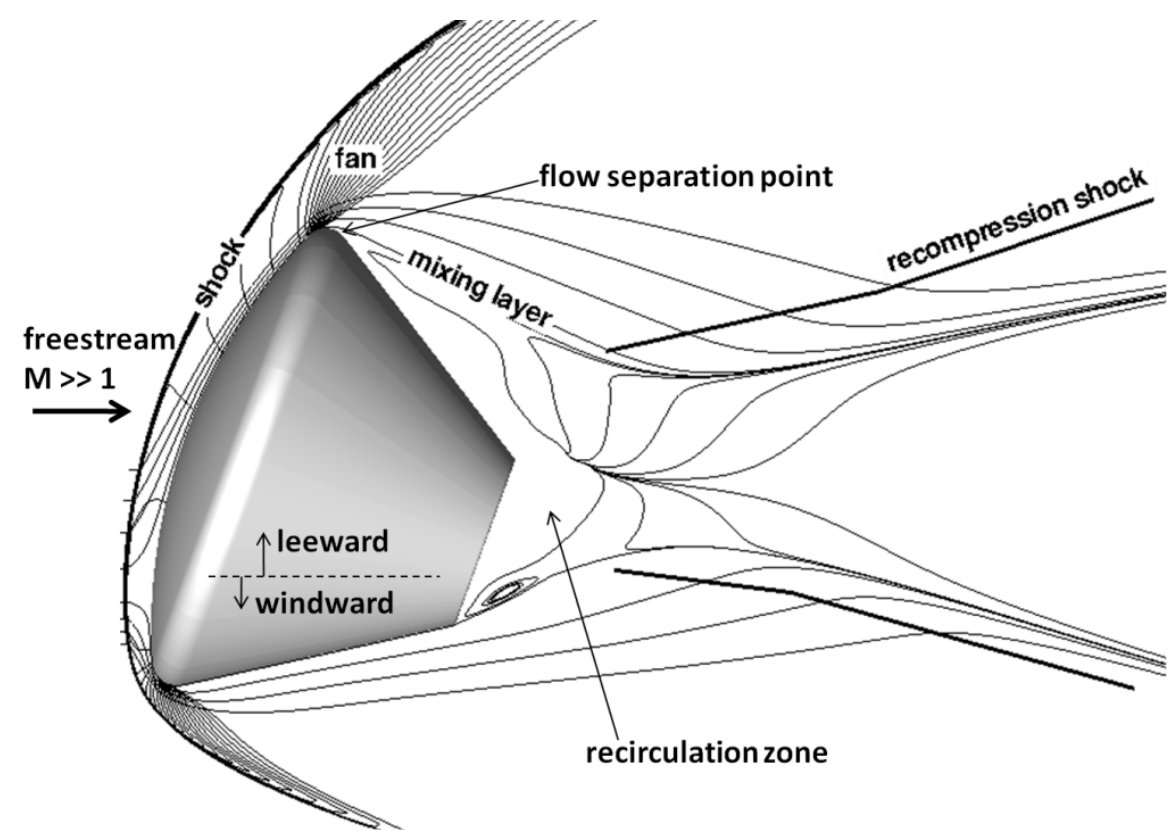

Figure 2. Schematic of a capsule-type reentry vehicle in a hypersonic flow field (adapted from Ref. 13). Black lines indicate shock waves and streamlines as well as pressure iso-bars. The iso-bars show the area of expansion around the leeward and windward cone shoulders.

In addition to the aforementioned flow structures around a blunt body shape, the presence of RCS jets on the vehicle's aftbody adds another layer of complexity to the flow. Although the jets interact with both the wake flow and with each other, studying an isolated jet in crossflow can aid in the interpretation of the results presented later. Figure 3 shows side and isometric views of underexpanded jets in supersonic crossflows, obtained from the literature. ${ }^{18,19} \mathrm{~A}$ bow shock wave forms ahead of the jet due to the low streamwise momentum of the jet fluid relative to the crossflow. The interaction of the bow shock wave with the upstream boundary layer causes a the flow to separate forward of the bow shock. Downstream of the bow shock wave, spanwise vorticity in the boundary layer rolls up around the jet, forming a horseshoe vortex. At the lip of the jet orifice, Prandtl-Meyer expansion occurs due to the large jet-to-crossflow pressure ratio resulting in an underexpanded jet. The jet gas is subsequently compressed by a barrel shock wave and later by a Mach disk. A shear layer forms between the barrel shock wave and the bow shock wave, which provides the mechanism for the production of turbulent eddies. A counter-rotating vortex pair is shown in the isometric view of Fig. 3. The vortex pair interacts with the boundary layer and convects high momentum, high temperature gas from the freestream down to the wall surface.

(a)

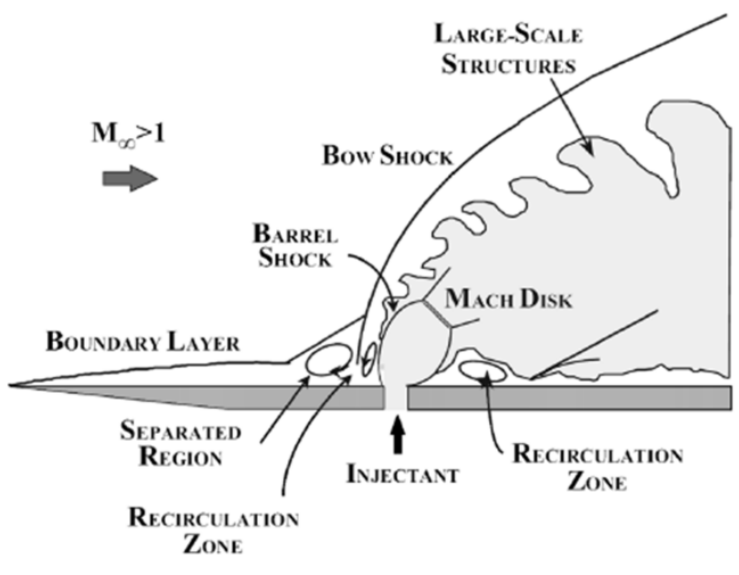

(b)

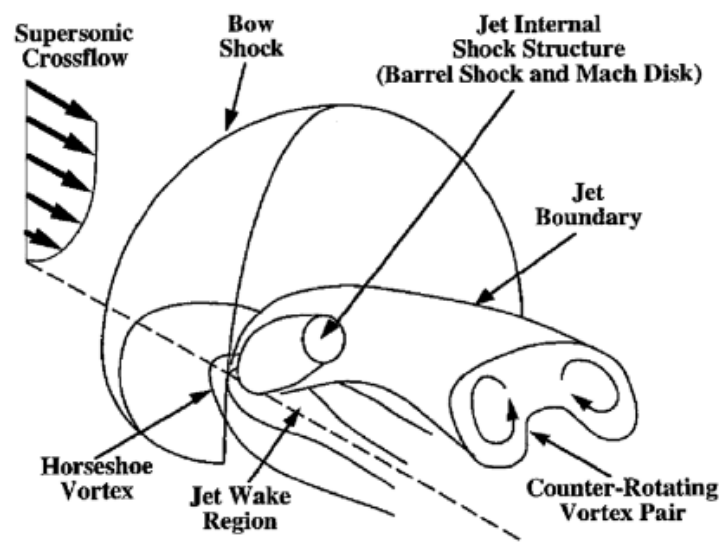

Figure 3. Schematics of an underexpanded supersonic jet in crossflow. The side-view (a) is an instantaneous snapshot of the flow field (Ref. 18). The isometric view (b) is a time-averaged sketch of the flow field (Ref. 19). 


\section{Experimental Results and Comparison with CFD}

\section{A. PLIF Flow Visualization}

Figures 4 and 5 show a summary of the NO PLIF flow visualization data set obtained in the wind tunnel tests. They compare pitch, roll and yaw jet flows for low tunnel stagnation pressure (Fig. 4) and high tunnel stagnation pressure (Fig. 5) cases. While these results are qualitatively similar to those described in detail in Ref. 11, these images were obtained with a slightly different Orion model configuration in a different test entry. (In the current paper, the Orion configuration is 606D and the test number was Test 455). The main focus of this paper is the comparison between the CFD computations and the fluorescence imaging and velocimetry for the yaw jet case. The experimental results in Figs. 4 and 5 will therefore only be briefly described.

(a1)

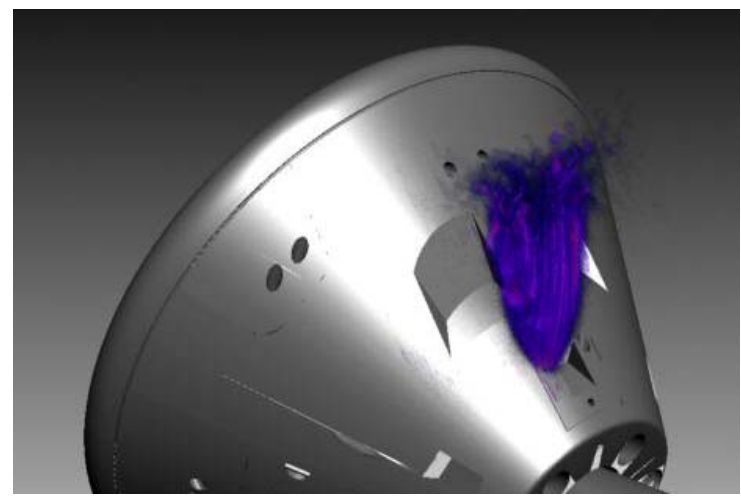

(a2)

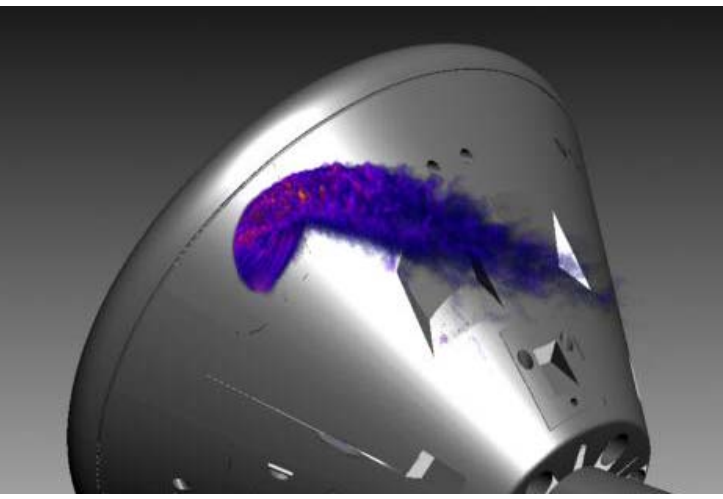

(a3)

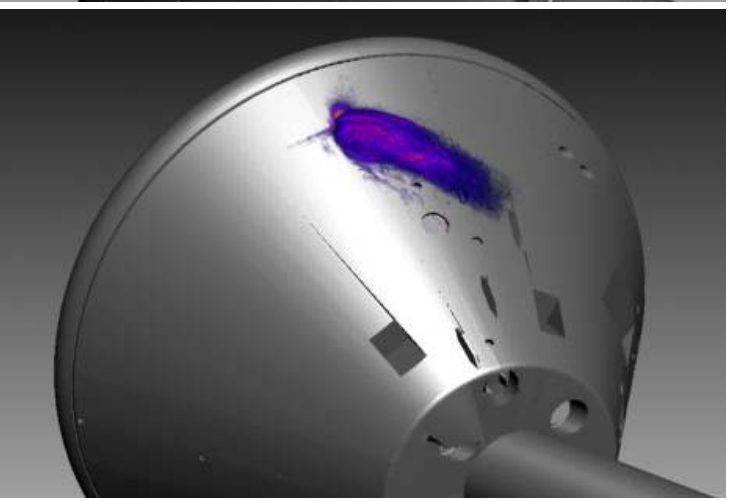

(b1)

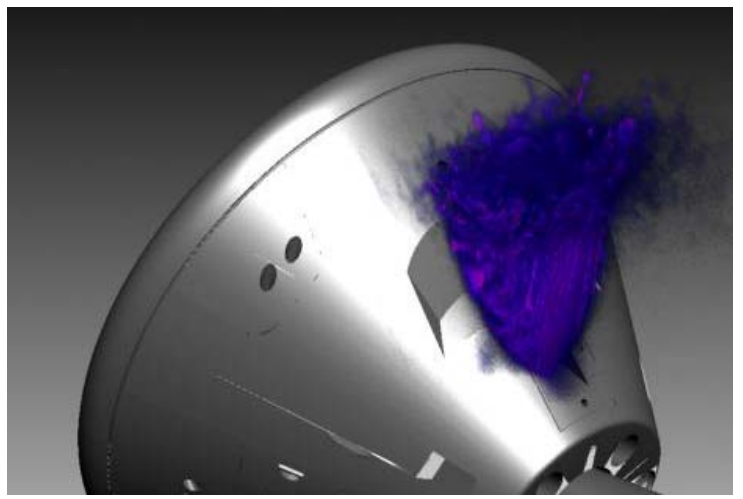

(b2)

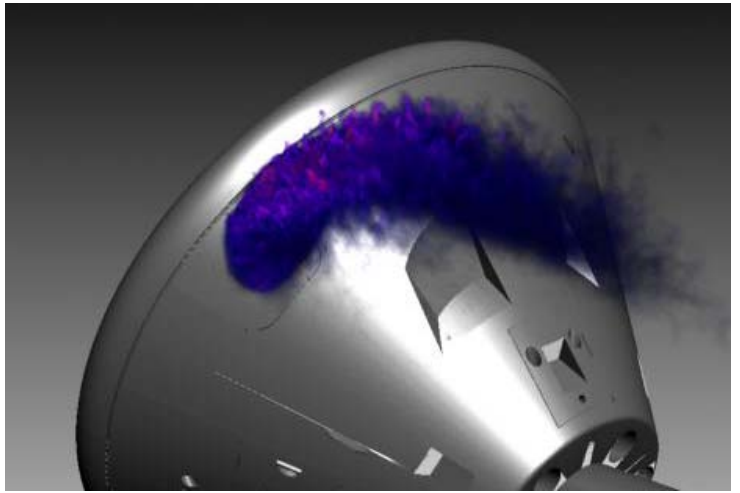

(b3)

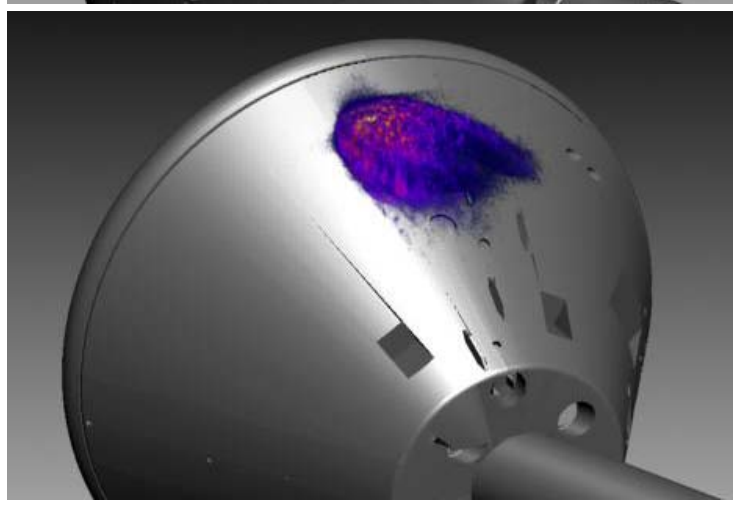

Figure 4. Isometric views of experimental PLIF volumetric flow visualizations with $\mathbf{P}_{\mathbf{0}}=350 \mathrm{psi}$. Column (a) has $P_{c} \sim 45$ psi. Column (b) has $P_{c} \sim 132$ psi. Figures (a1) and (b1) show the pitch jet; (a2) and (b2) show the roll jet; (a3) and (b3) show the yaw jet (note model rotation $90^{\circ}$ about the sting in the third row relative to the first two rows). 

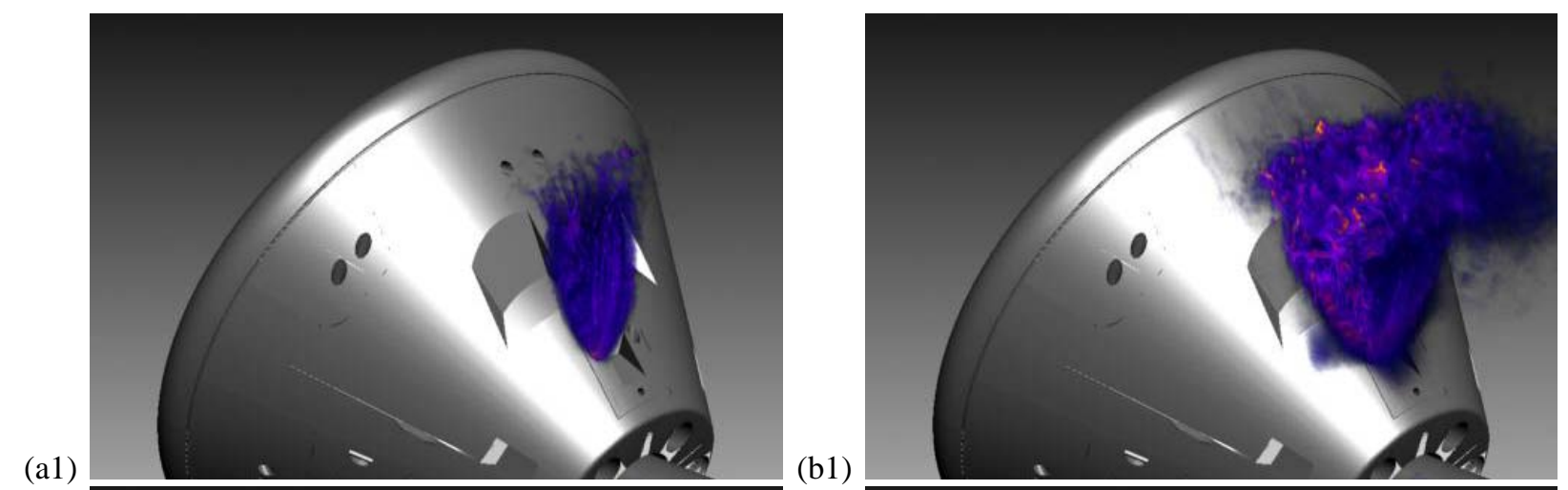

(a2)

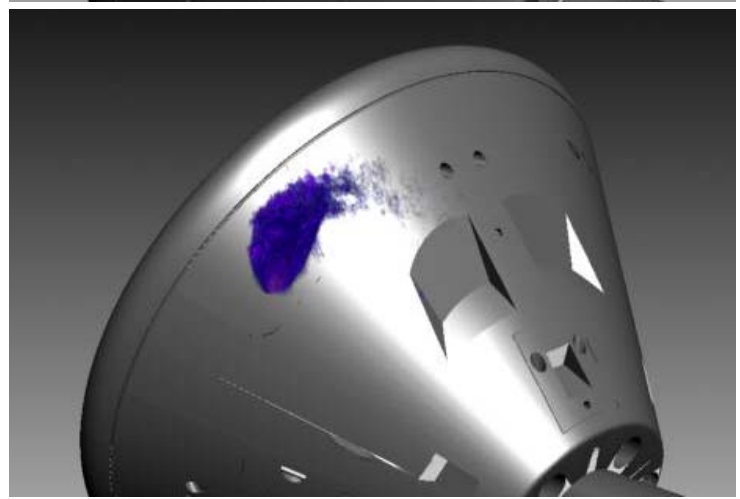

\section{(b2)}

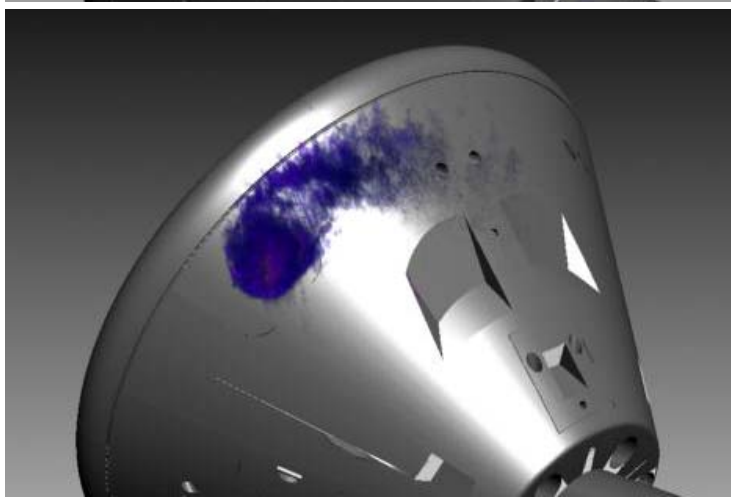

(a3)
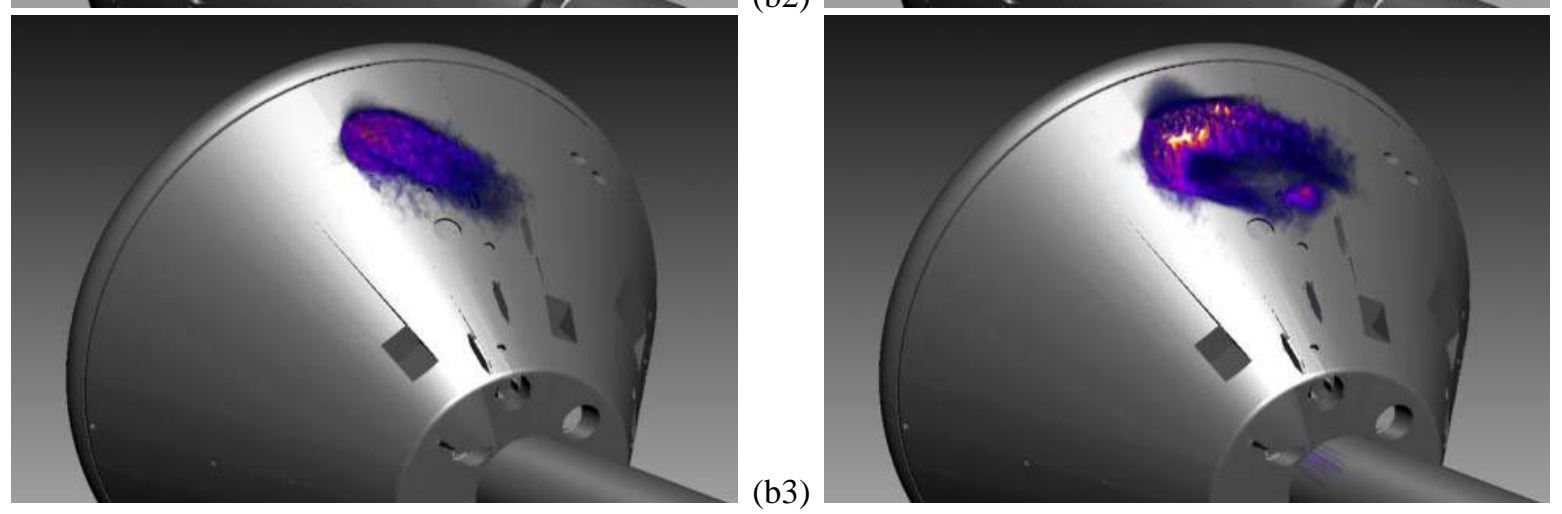

Figure 5. Isometric views of PLIF volumetric flow visualizations with $P_{0}=1,300$ psi. Column (a) has $P_{c} \sim 132$ psi. Column (b) has $P_{c} \sim 425$ psi. Figures (a1) and (b1) show the pitch jet; (a2) and (b2) show the roll jet; (a3) and (b3) show the yaw jet (note model rotation).

In the top row of Fig. 4, the pitch-up jets issue into the centerline wake flow region, which is a large separated region having relatively low static pressure. Near the jet exit, there exists a large separated flow region, containing slowly moving gas, resulting in very weak crossflow. Strong fluorescence signal continues along the jet's trajectory until the jet reaches the shear layer between the separated wake flow region and the flow passing over the heat shield and over the shoulder of the model. The jet fluid is then observed to flow mostly downstream, though some jet fluid impinges on the aft-body thermal protection system as well. The roll jets, in the middle row of Fig. 4, initially propagate vertically (nearly azimuthally) out of the jet exit. When the jet fluid reaches the oncoming crossflow passing around the side of the model, the jets are deflected downstream towards the sting. The roll jets appear to partially form the counter-rotating vortices shown in Figs. 2 and 3. To study the yaw jet, the model was rotated $90^{\circ}$ counter clockwise (as viewed from upstream) about the sting. The yaw jet visualizations shown in the bottom rows of Figs. 4 and 5 also have been rotated by $90^{\circ}$ compared to the pitch and roll visualizations. Considering cases with similar jet chamber pressures, $P_{c}$, the pitch jets appear largest in diameter and the yaw jets appear smallest. These trends are caused by the spatial pressure distribution on the backshell as well as the varying size of the separated flow region at the different jet 
locations. Higher pressures and smaller separated regions reduce the extent of the jet flow. The higher $P_{c}$ in the rightcolumn figures results in a larger diameter jet, evident in pitch, roll and yaw jets.

In Fig. 5, the stagnation pressure, $P_{0}$, increases by a factor of 3.7 while the values of $P_{c}$ increase by about a factor of 3 from the corresponding cases in Fig. 4. Thus the ratio of $P_{d} P_{o}$ stays roughly constant in Figs. 4 and 5 . The main factor that changes is the Reynolds number, with Fig. 5 having a 3.7 times higher Reynolds number than Fig. 4. In comparing these two cases, significant changes in the flow features (size, shape, trajectory of the jets) are not observed. The fluorescence intensity decreases more quickly with downstream distance, for example in the roll-jet cases, but this is probably because the higher pressure results in faster quenching of the NO fluorescence and consequently lower signal. This does not imply any fluid mechanical significance. One would expect more turbulent flow features in the higher Reynolds number cases, but the lower Reynolds number case already appears rather turbulent at the edges of the jets observed with this type of volumetric visualization. Numerous other views including single-plane images and animations of these flow visualizations have been produced and are available from the authors.

\section{B. PLIF Velocity Measurements}

Figure 6 shows the average experimental velocities and measurement uncertainties for two chamber pressures, $P_{c}$, obtained during the same run. The lower chamber pressure case generally has larger uncertainties than the higher chamber pressure case due to a smaller number of measurements averaged together. ${ }^{8}$ Generally, the uncertainties in the velocity increase with downstream distance and increase dramatically near the edge of the jet. The larger uncertainties near the boundary of the jet are due primarily to the unsteady nature of the flow features. Velocity data near the jet exit were removed due to a systematic error in the velocity calculation caused by a large, negative quenching gradient over the width of the line. ${ }^{8}$

(a)

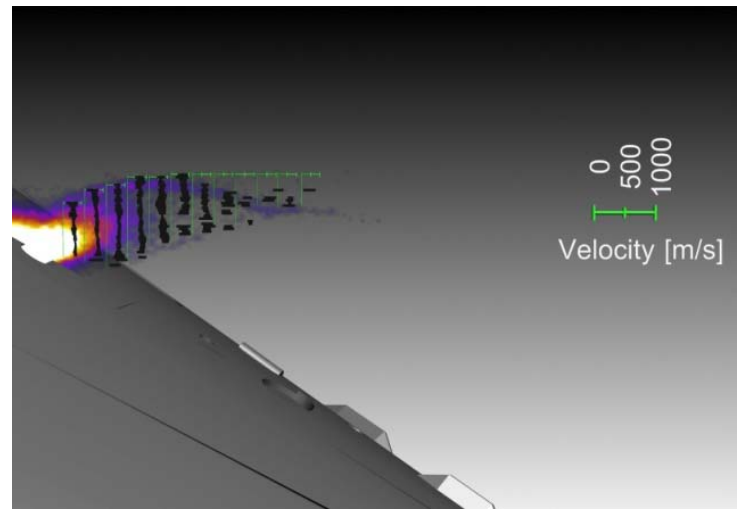

(b)

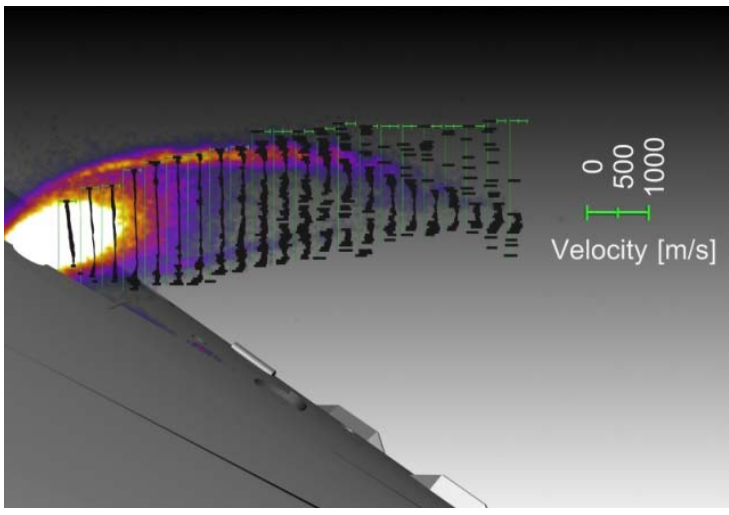

Figure 6. PLIF MTV yaw jet measurements with $P_{0}=350$ psi. The position of the center of the black line relative to its local green scale bar indicates its mean velocity. The thickness of the black line relative to its local scale bar indicates the uncertainty in the velocity measurement. Figure (a) has $P_{c} \sim 45$ psi; (b) has $P_{c} \sim 132$ psi.

\section{CFD Temperature, Density, Pressure, and Velocity}

The CFD temperature, density, pressure, and streamwise velocity maps for yaw jet operation shown in Fig. 7 are all taken in the plane passing through the jet centerline position and oriented in the vertical plane, parallel to the streamwise direction. The temperature images in Fig. 7 show several of the flow features described in Figs. 2 and 3. The smooth change in temperature caused by the expansion fan from the shoulder of the model is observed in the top left corner of each image. This high speed gas then interacts with the jet plume, resulting in an oblique bow shock, observed as a diagonal high-temperature region in Figs. 6(a) and (b). Some unphysical oscillations are observed in this bow shock, as well as in the downstream jet plume. These artifacts may be caused by non-optimal grid density and alignment in the wake region. ${ }^{13}$ The post-shock gas cools as it mixes with the cold RCS jet fluid, which has a stagnation temperature of approximately room temperature and has been expanded past Mach 4. A room-temperature stagnant region exists towards the bottom-right side of both Figs. 6(a) and (b). The computed density, pressure and velocity presented in Fig. 6 corroborate with this description and are qualitatively similar to many (time-averaged) aspects of Figs. 2 and 3. 


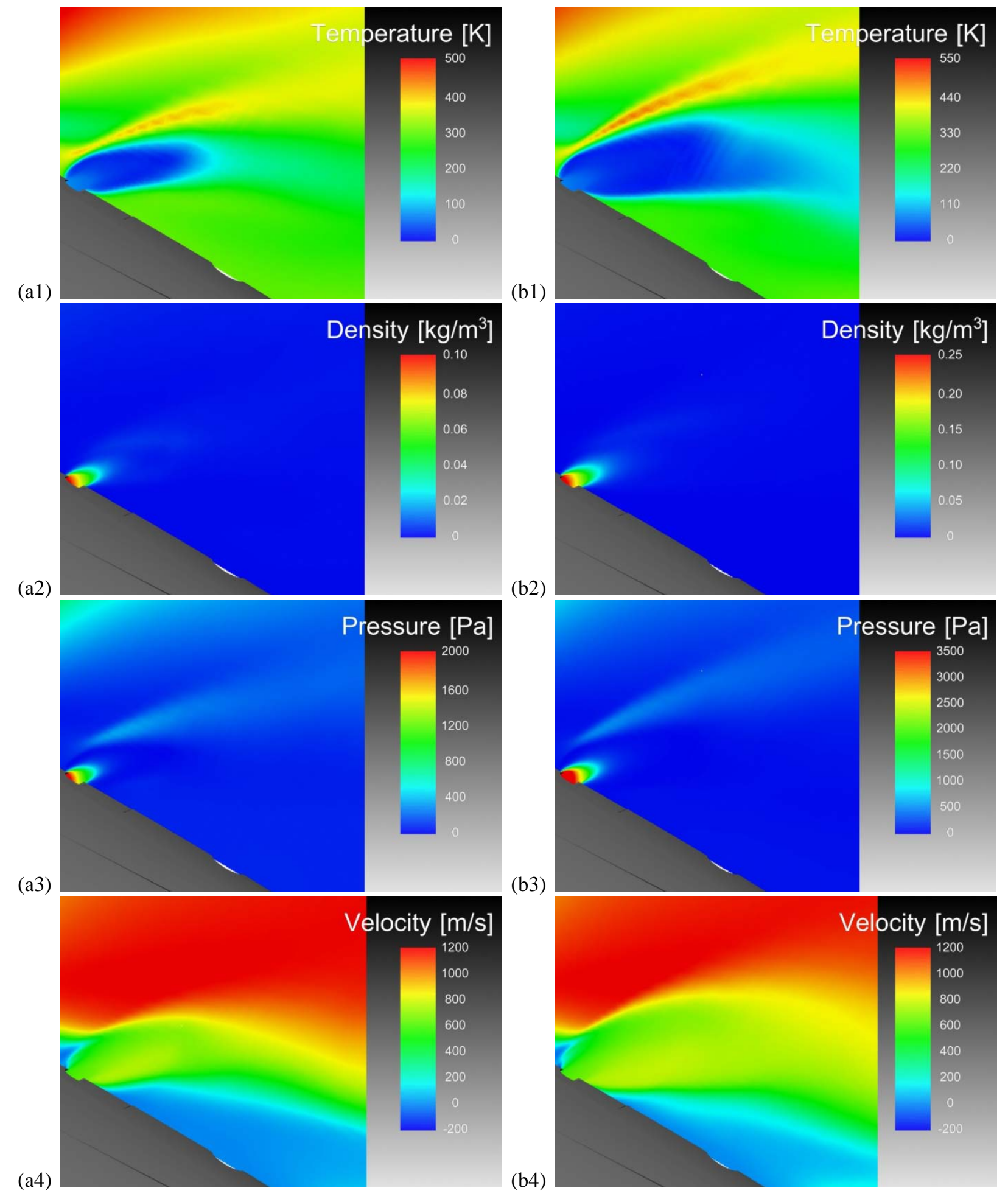

Figure 7. CFD temperature, density, pressure, and streamwise velocity results with $\mathbf{P}_{0}=350$ psi. Column (a) has $P_{c} \sim 45$ psi. Column (b) has $P_{c} \sim 132$ psi. 
D. CFI and PLIF Flow Visualization Comparison

Figures 8 and 9 compare theoretical (CFI) and experimental PLIF fluorescence images for the yaw Orion RCS jet.

(a1)

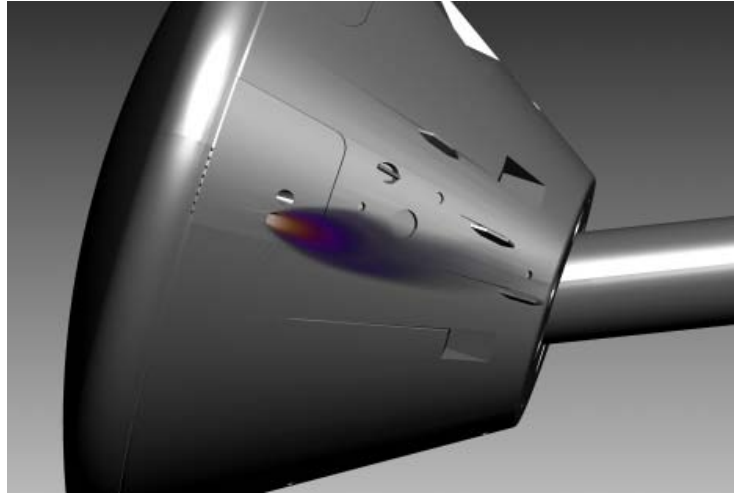

(a2)

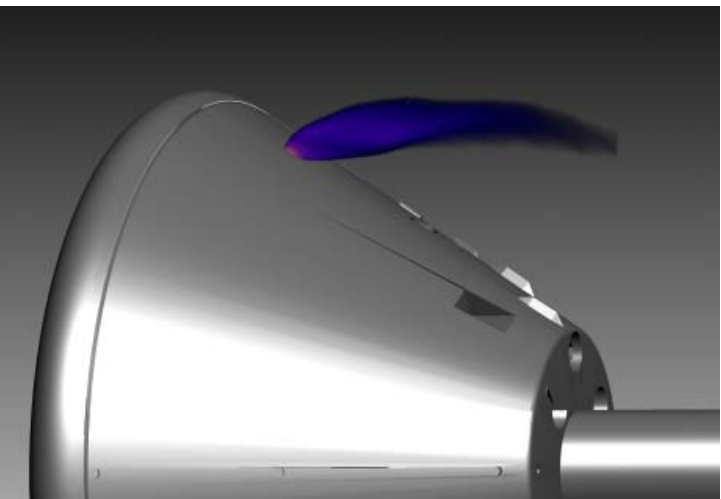

(a3)

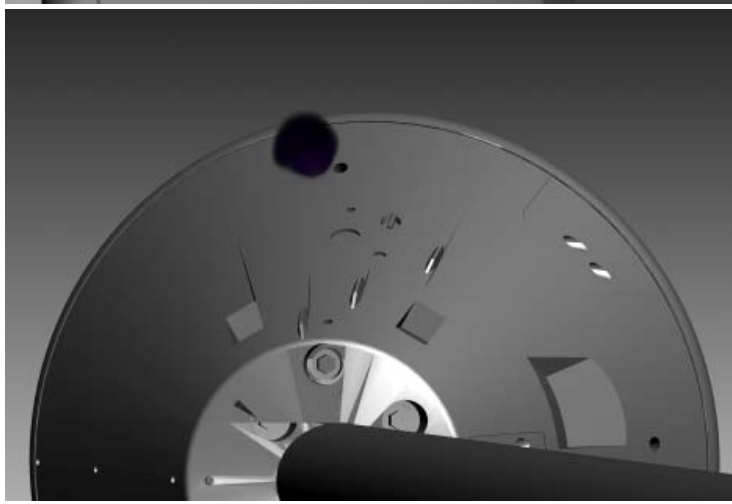

(a4)

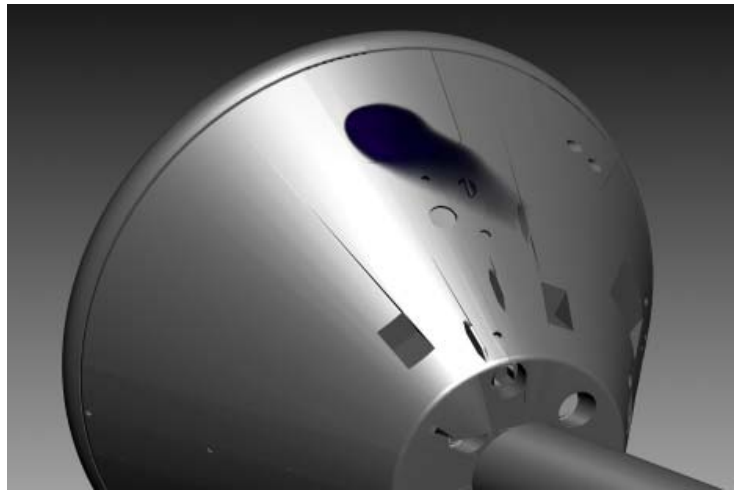

(b1)

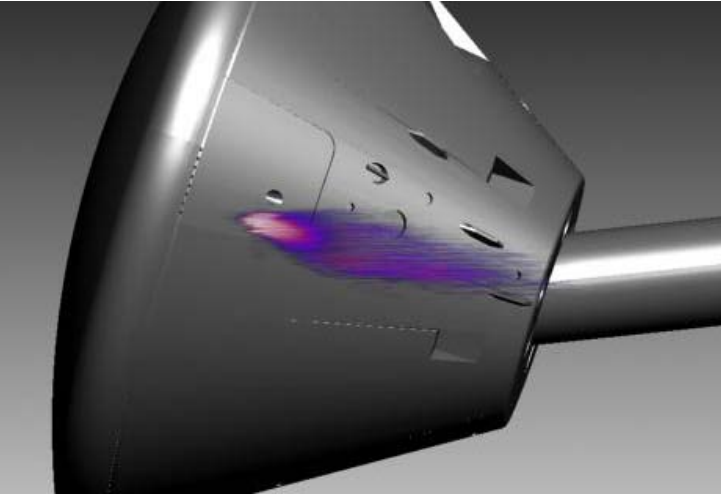

(b2)

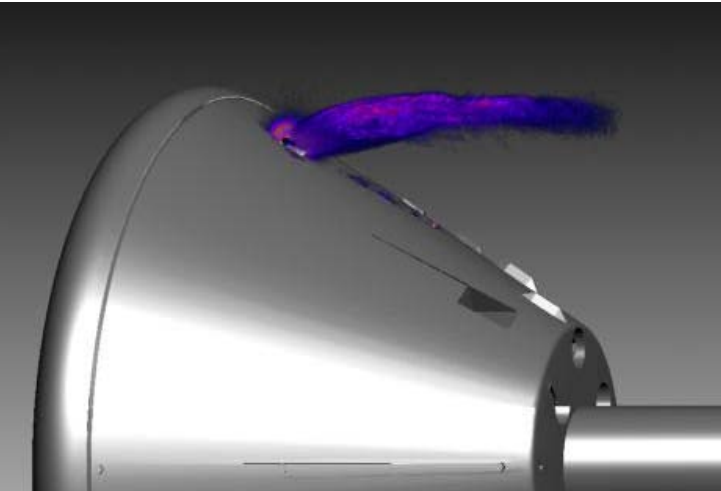

(b3)

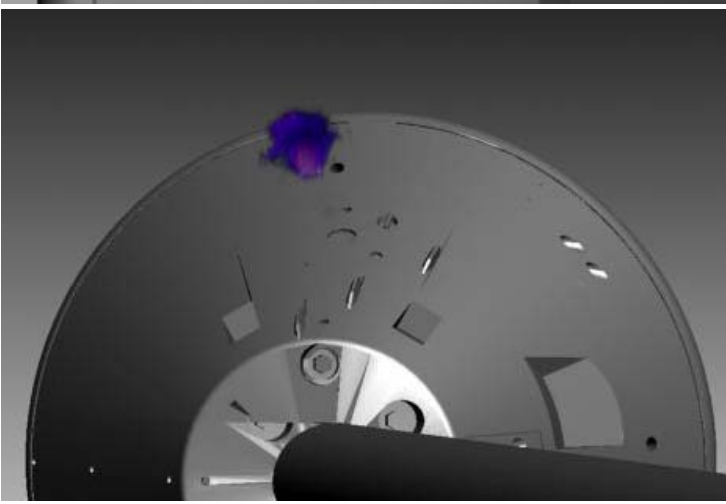

(b4)

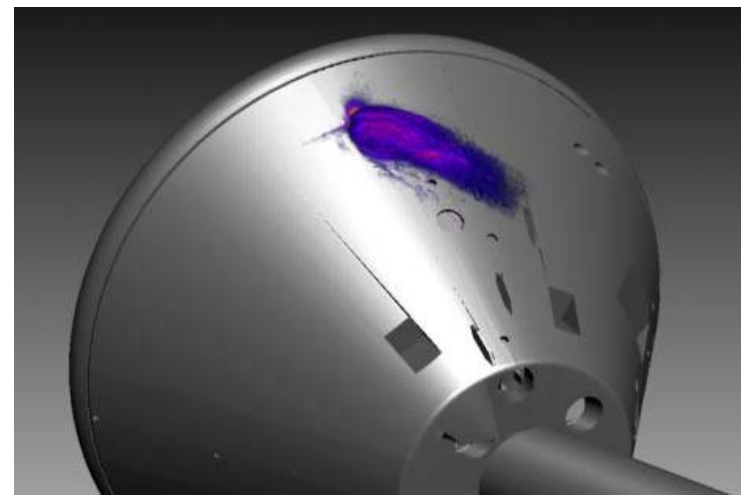

Figure 8. CFI and Experimental PLIF yaw jet flow visualizations with $P_{0}=350$ psi and $P_{c} \sim 45$ psi. Column (a) shows the CFI images. Column (b) shows the comparable PLIF flow visualization. (a1) and (b1) are side views; (a2) and (b2) are bottom views; (a3) and (b3) are back views; (a4) and (b4) are isometric views. 

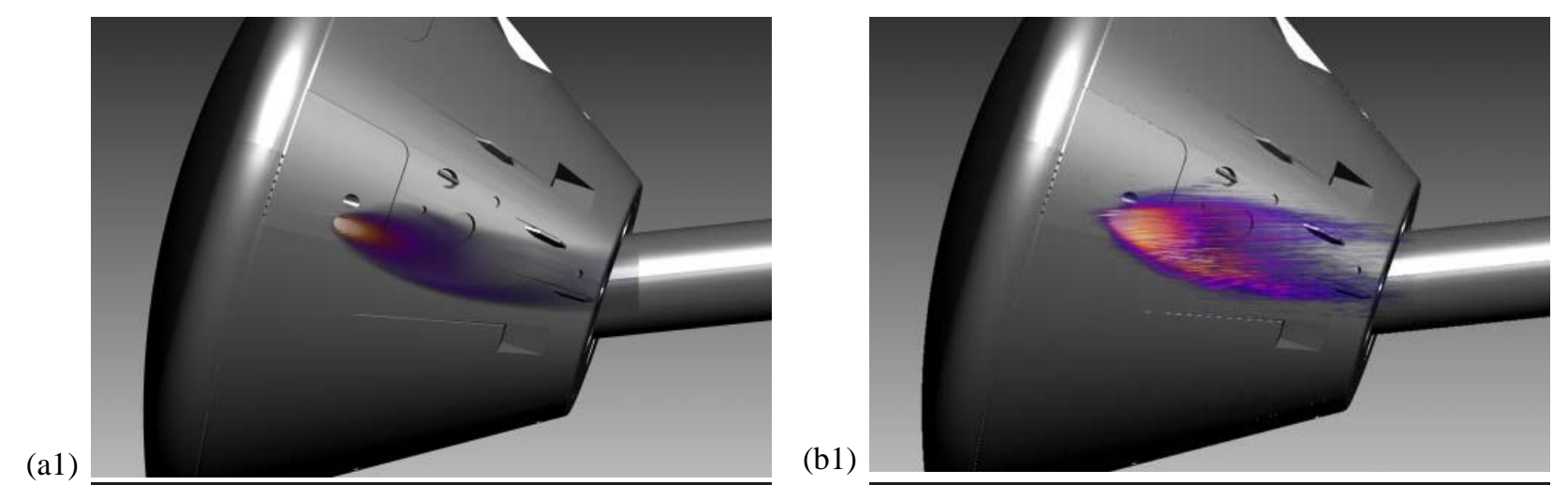

(a2)

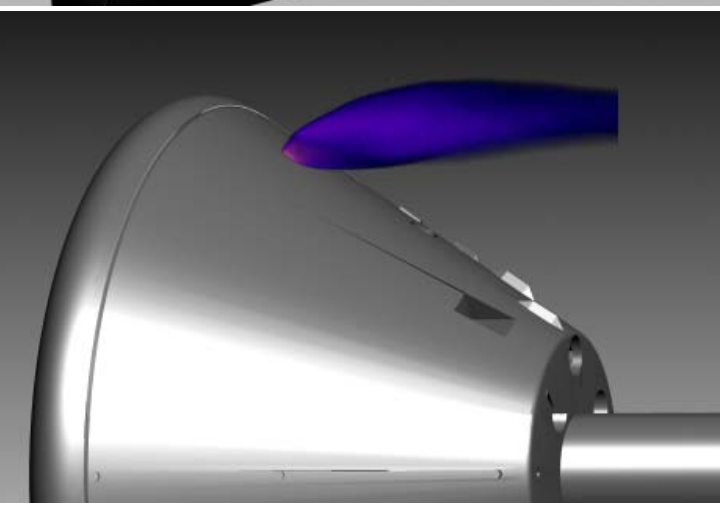

(a3)

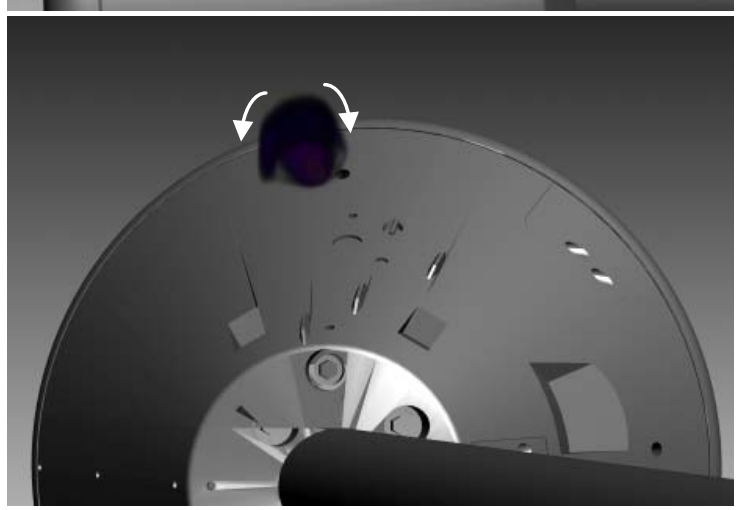

(b2)

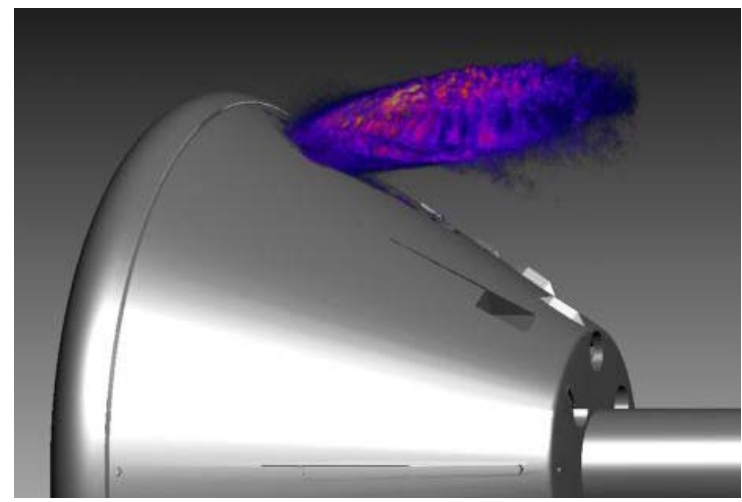

(b3)

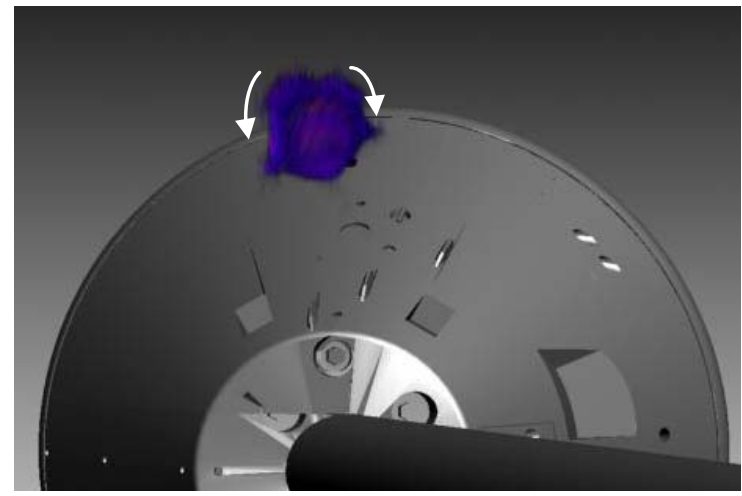

(a4)
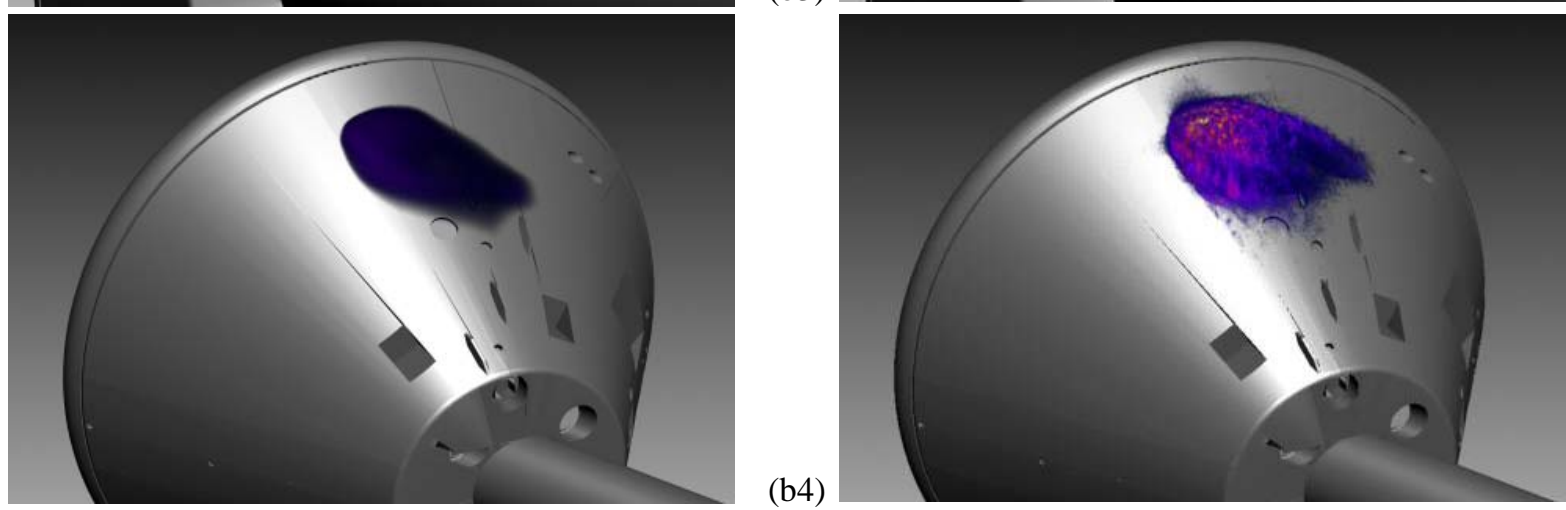

Figure 9. CFI and Experimental PLIF yaw jet flow visualizations with $P_{0}=350$ psi and $P_{c} \sim 132$ psi. Column (a) shows the CFI images. Column (b) shows the comparable PLIF flow visualizations. (a1) and (b1) are side views. (a2) and (b2) are bottom views. (a3) and (b3) are back views. (a4) and (b4) are isometric views.

The comparison in Fig. 8 and 9 is made by simultaneously displaying images from many closely spaced vertical slices through the yaw jet. The images show that the CFI is able to convey very similar three-dimensional geometries of the yaw RCS jet for both of the chamber pressures. The 'back' views in the third row of both figures show the expected 
counter-rotating vortices forming on the edge of the jet as in a classical jet in a crossflow, shown in Fig. 3(b). These are highlighted by arrows in Fig. 9(a3) and (b3). The darker appearance of the CFI PLIF in the bottom two rows of each figure is not fluid-mechanically significant. It is an artifact of the color table used to display the images and the spacing of the CFI-PLIF planes. An obvious difference between the CFI and experimental plumes is that the CFI plumes are smooth, owing to the laminar nature of the computation, while the experimental data show turbulent structures on the edges of the jets (though they also show laminar flow in the core of the jets, as shown in Fig. 10). The origin of these turbulent structures on the outer edges of the jet is described above, in the explanation of Fig. 3(a).

(a1)

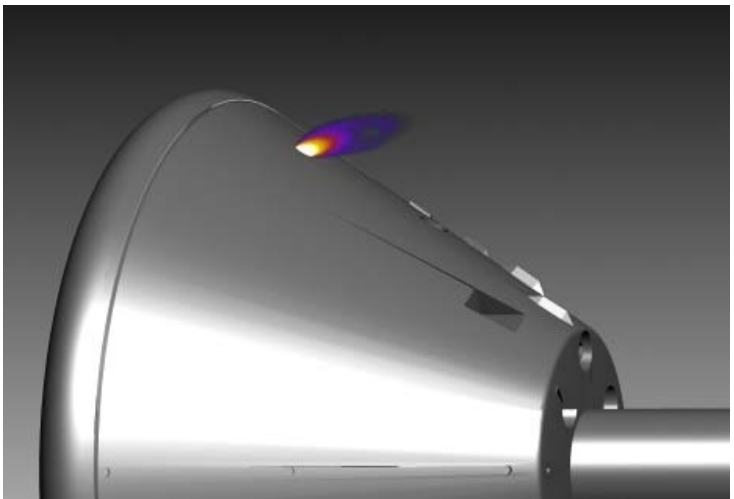

(a2)

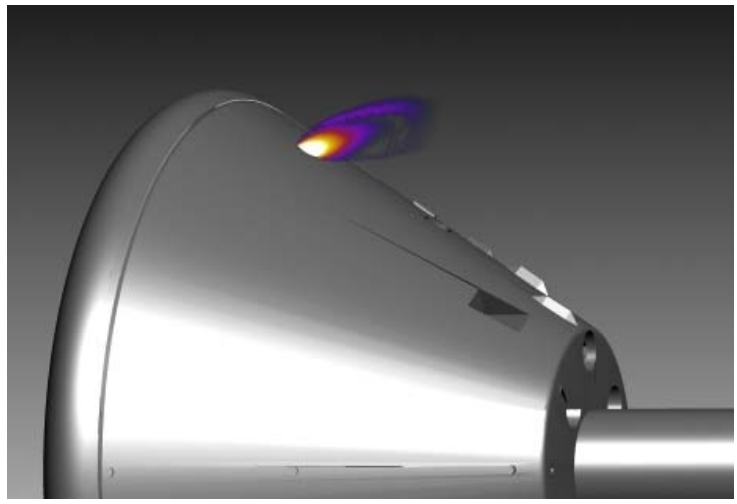

(b1)

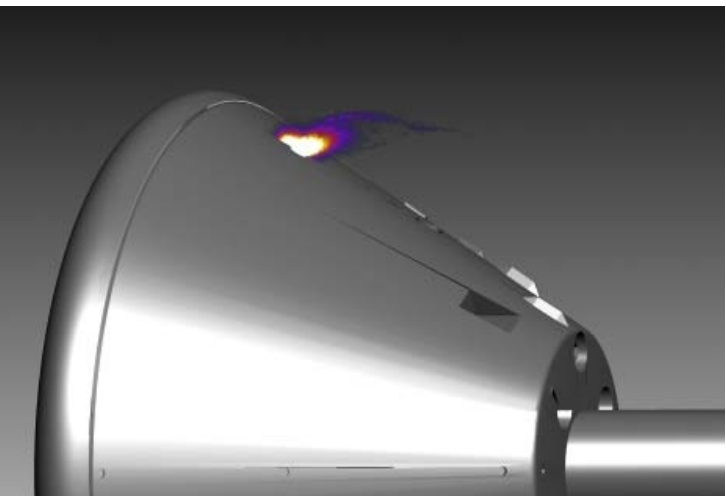

(b2)

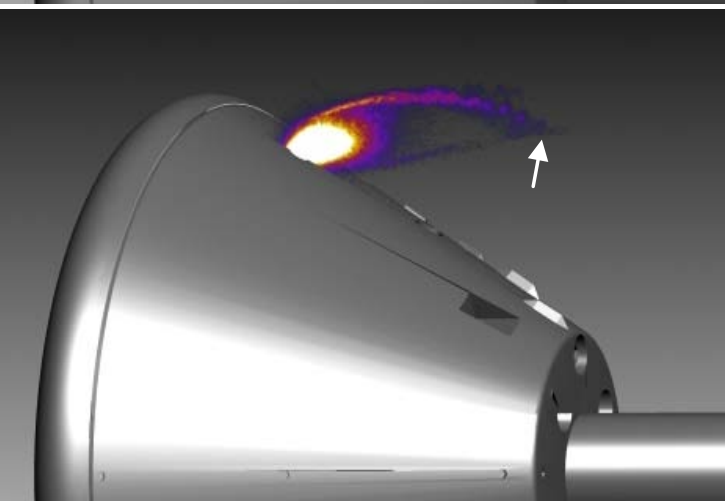

Figure 10. CFI and Experimental PLIF yaw jet flow visualizations with $\mathbf{P}_{0}=350$ psi. Column (a) shows the CFI images. Column (b) shows the comparable PLIF flow visualizations. (a1) and (b1) are bottom views with $P_{c} \sim 45$ psi. (a2) and (b2) are bottom views with $P_{c} \sim 132$ psi.

Whereas Figs. 8 and 9 show the three-dimensional structure of the yaw jet by displaying data from multiple planes simultaneously, Fig. 10 shows some of the finer structures of the jet as revealed by single image planes. The CFI and PLIF centerline images, shown in Fig. 10, illustrate some discrepancies between theory and experiment. The CFI has a more diffuse and smaller sized barrel-shock boundary. The furthest downstream extent of the jet, where one might expect a Mach disk to appear, shows a rounded shape in the CFI while the experimental image shows a pointed structure, indicated by the arrow in Fig. 10(b2). The presence of barrel shock waves and Mach disk boundaries are expected in the PLIF images since sudden changes in pressure and temperature are closely related to changes in fluorescence (Eq. 10). Some small, unphysical diagonal features are observed inside the barrel shock in Fig. 10(a2). These may be grid alignment artifacts, ${ }^{13}$ suggesting that additional refinements to the grid might help improve the comparison between theory and experiment. 


\section{E. CFI Representation Comparison}

Figure 11 shows the results of a comparative study conducted on the center plane of the yaw jet flow visualization test case with $P_{0}=350 \mathrm{psi}$ and $P_{c} \sim 132 \mathrm{psi}$. Figure 11 compares two different NO seeding concentrations as well as two different ways to compute and display the CFI PLIF images. Figure 11(a1) was computed assuming that the spectral overlap integral, $g$, is Gaussian-Voigt (a Gaussian laser profile and a Voigt absorption profile) and the mole fraction of nitric oxide was $\chi_{N O}=0.04$; Fig. 11(b1) assumes $g$ is triple-Gaussian and $\chi_{N O}=0.04$; Fig. 11(a2) assumes $g$ is triple-Gaussian and $\chi_{N O}=0.005$; and Fig. 11(b2) uses the $S_{f \text {, wilkes }}$ representation. Figure (a3) is a line plot taken approximately down the centerline of the jet, downstream of the jet exit, showing relative intensities for the CFI representations in Figs. 11(a1), 11(b1), 11(a2), and 11(b2). The fluorescence profiles in Fig. 11(a3) are scaled relative to each other to approximately match the fluorescence intensity downstream. Also shown in Fig. 11(a3) is a cutoff for the $S_{f, \text { wilkes }}$ representation used to saturate the jet exit and increase the surrounding intensity, as shown Fig. 11(b3). Figures 11(a1) and 11(b1) look nearly identical, demonstrating that the triple-Gaussian spectral representation approximated the Gaussian-Voigt profile very well. This is a significant result because it means that the convolution integral in Eq. 6 does not have to be computed. This reduces the computational time by 2-3 orders of magnitude (depending on the number of integration increments used). This comparison is also shown more clearly in Fig. 11(a3) where a single profile through the center of the jet is shown.

(a1)

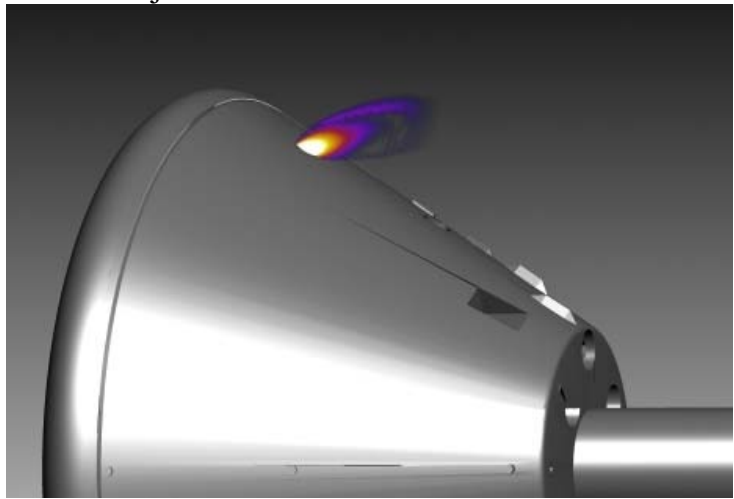

(a2)

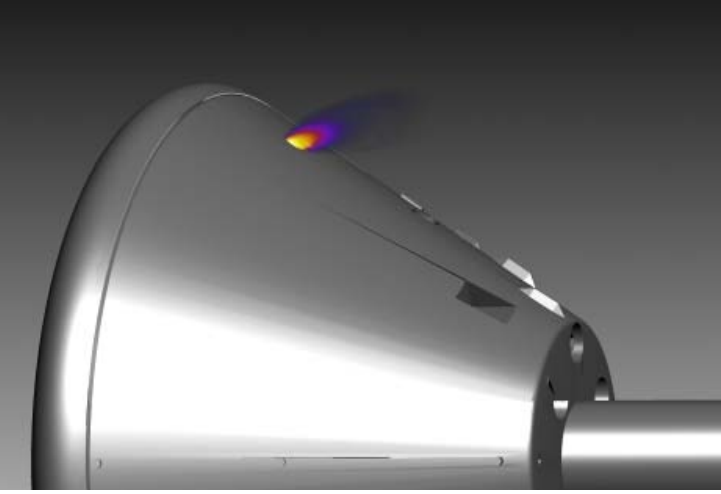

(a3)

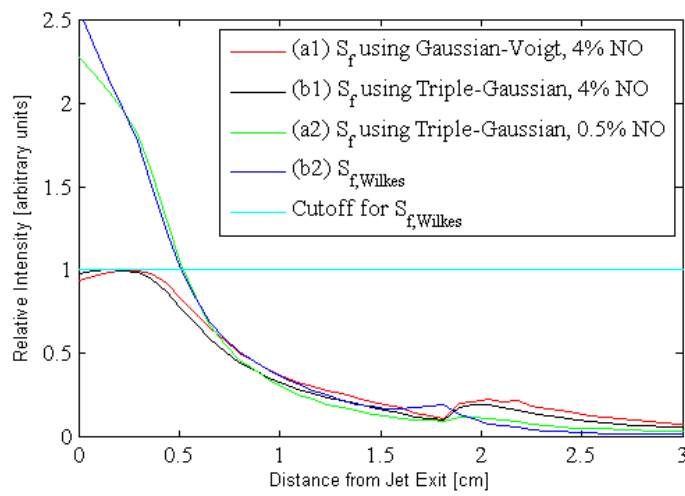

(b1)

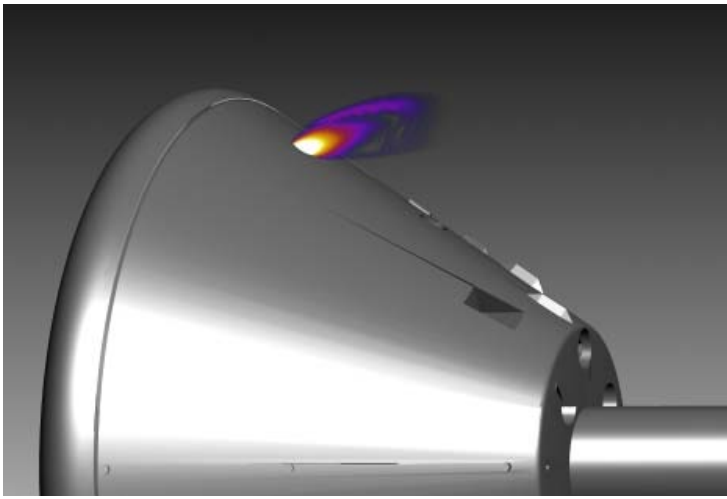

(b2)

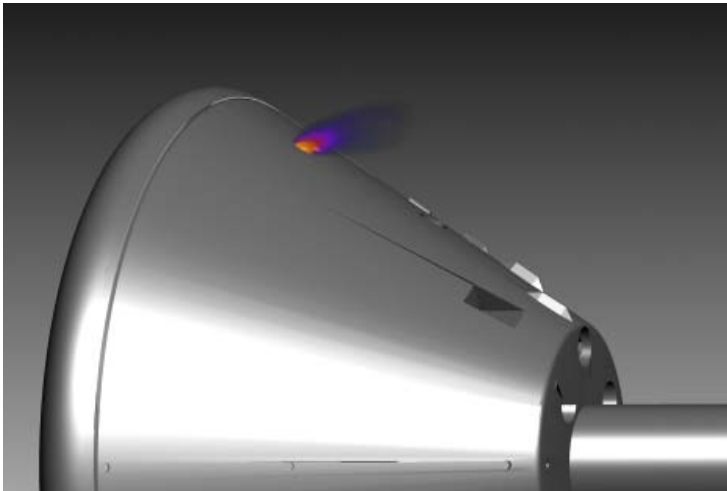

(b3)

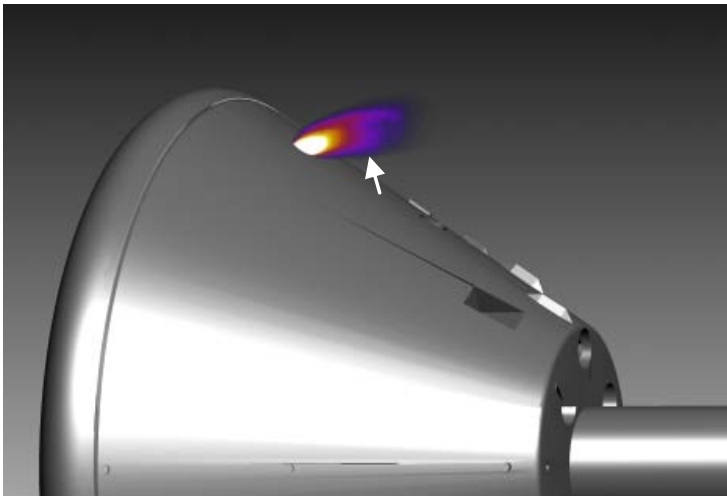

Figure 11. CFI yaw jet flow visualizations and line plot with $P_{0}=350$ psi and $P_{c} \sim 132$ psi. (a1), (b1), (a2), (b2) and (b3) are CFI images of the same fluid domain using different assumptions, explained in the text. (a3) is a line graph comparing how the different models indicate the same profile down the center of the jet. 
Figures 11(a2) and (b2), computed with nearly a factor of 10 lower NO seeding, though identical overall jet mass flow rate, show a more diffuse and slightly smaller-appearing jet boundary than the higher seeding cases in Fig 11(a1) and (b1). The apparent decrease in jet size is probably due to the color table, which is scaled to the maximum value in the image: higher peak values near the jet exit make the jet appear narrower (the CFD computation was the same for both figures). Figures 11(a2) and (b2) show that the simple $S_{f \text {, wilkes }}$ expression computes a PLIF distribution (b2) that is very close to the more sophisticated triple-Gaussian computation in (a2). The fluorescence profiles shown in Fig. 11(a3) more clearly illustrate the effect of using a smaller concentration of NO. At lower concentrations, the fluorescence intensity increases dramatically near the jet exit, where there is the highest pressure and highest concentration of NO. At higher NO concentrations and higher pressures, the self-quenching of NO reduces the intensity near the jet exit. A drawback of using the lower NO concentration is that there is a very large change in signal between the jet exit and the downstream plume. So, if this concentration is used, the dynamic range of fluorescence intensity may be difficult to display on a linear color table as shown above (the jet exit will appear very bright and the downstream plume will not be visible). As a workaround, the $S_{f \text {, wilkes }}$ intensity can be purposefully saturated (in the color table) near the jet exit, for example at the relative fluorescence intensity shown by the cutoff in Fig. 11(a3), the results of which are shown in Fig. 11(b3). The CFI image presented in Fig. 11(b3) better shows the barrel shock boundary (as indicated by the white arrow) compared to 11(b2). The $S_{f \text {, wikes }}$ representation is much easier to implement and substantially faster than the representation of Eq. (1) assuming $g$ is triple-Gaussian, with time savings on the order of 2-3 magnitudes. Also, it would be a simple matter to compute a new version of the $S_{f \text {, wilkes }}$ expression for the higher seeding levels if desired. For future comparisons between computational and experimental PLIF images in RCS jet experiments, we recommend use of the simple $S_{f \text {, wikes }}$ expression. While it does not have all the physics embedded in the more complicated models, it is much easier to use, much faster, and can easily be implemented by a non-expert in Tecplot. Also, the level of discrepancies observed between the more sophisticated model and the experimental data are larger than the differences between $S_{f \text {, wikes }}$ and the more sophisticated models, at least for the RCS jets considered in the present study.

\section{F. CFD and MTV Velocity Comparison}

Experimental MTV measurements are shown overlaid on maps of CFD velocity computations for two different jet chamber pressures in Fig. 12(a1) and 12(b1). For each run condition, three measurement locations have been chosen for a more detailed comparison. In Figs. 12(a2)-12(a4) and 12(b2)-12(b4), lines showing the CFD results are plotted along with MTV data, including measurement uncertainty error bars, for these selected locations. As Fig. 12 illustrates, the CFD velocities generally showed similar growth and decay trends as the MTV measurements, with the theory generally fitting within the window of uncertainty of the measurement. Near the edges of the profile, the disparity between MTV and CFD velocities increased significantly. Also, the disparity between theory and experiment grows with distance downstream, as shown generally in Fig. 11(b1). In the future, the larger uncertainty bars could be reduced by a factor of 2-3 by averaging 4-9 times more measurements. This number of measurements (up to 900) could be obtained in a single tunnel run using the existing instrument. However, some uncertainties in the experiment ${ }^{8}$ will not be reduced by averaging. 

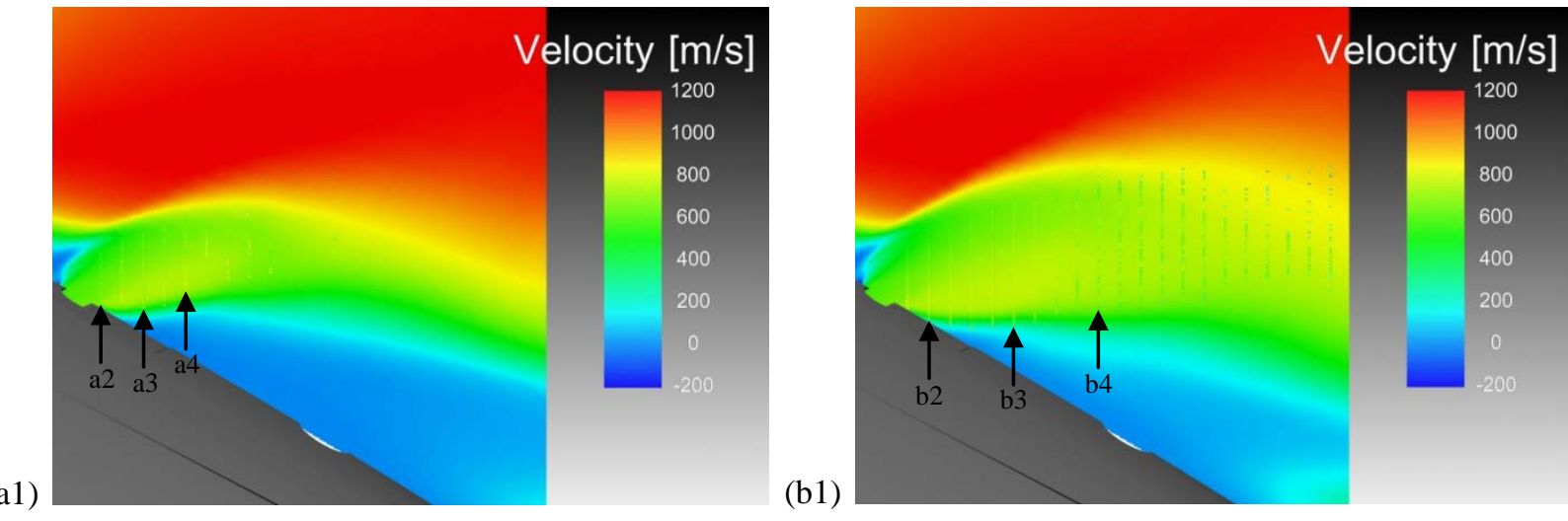

(a2)
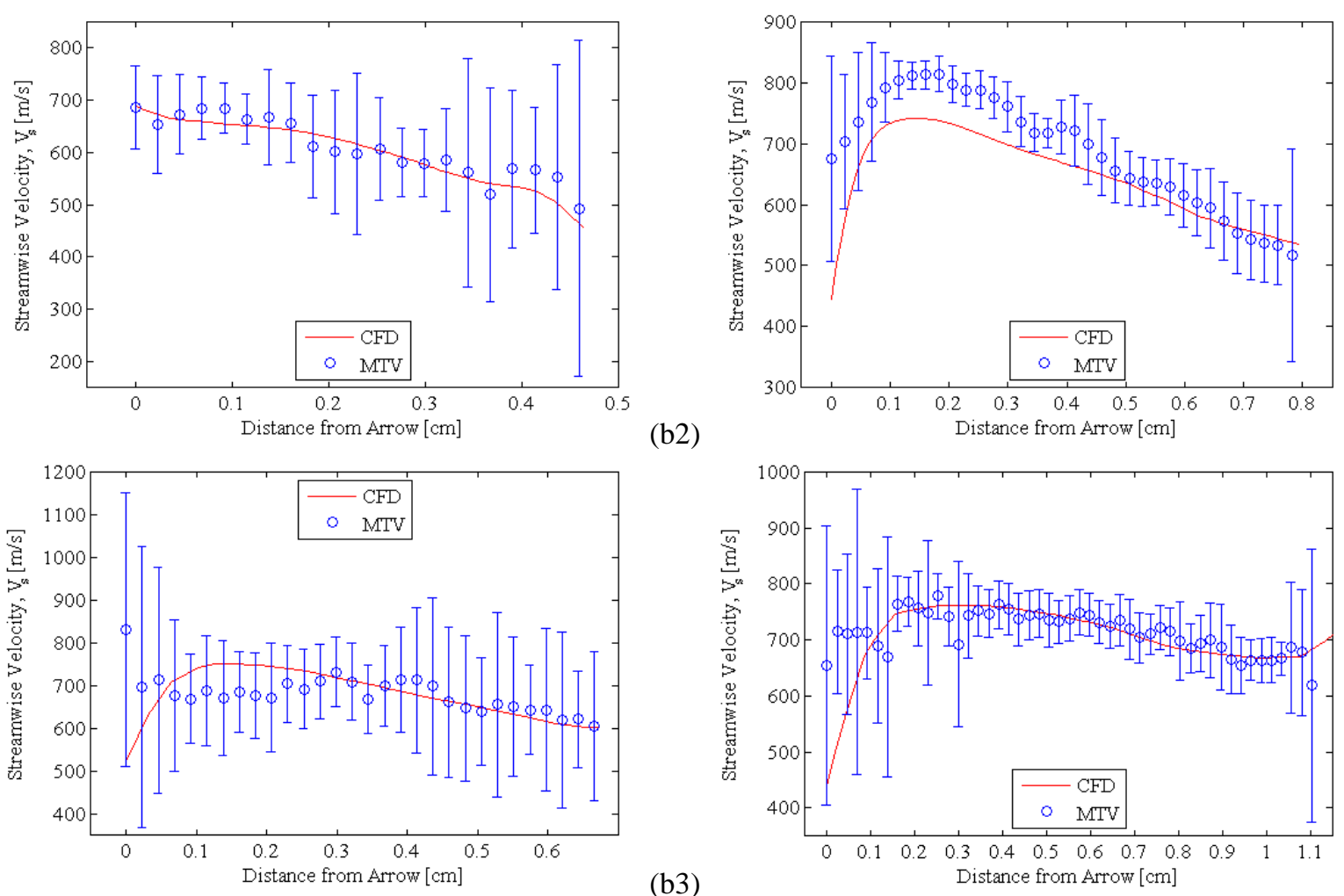

(b2)

(a3)

(b3)
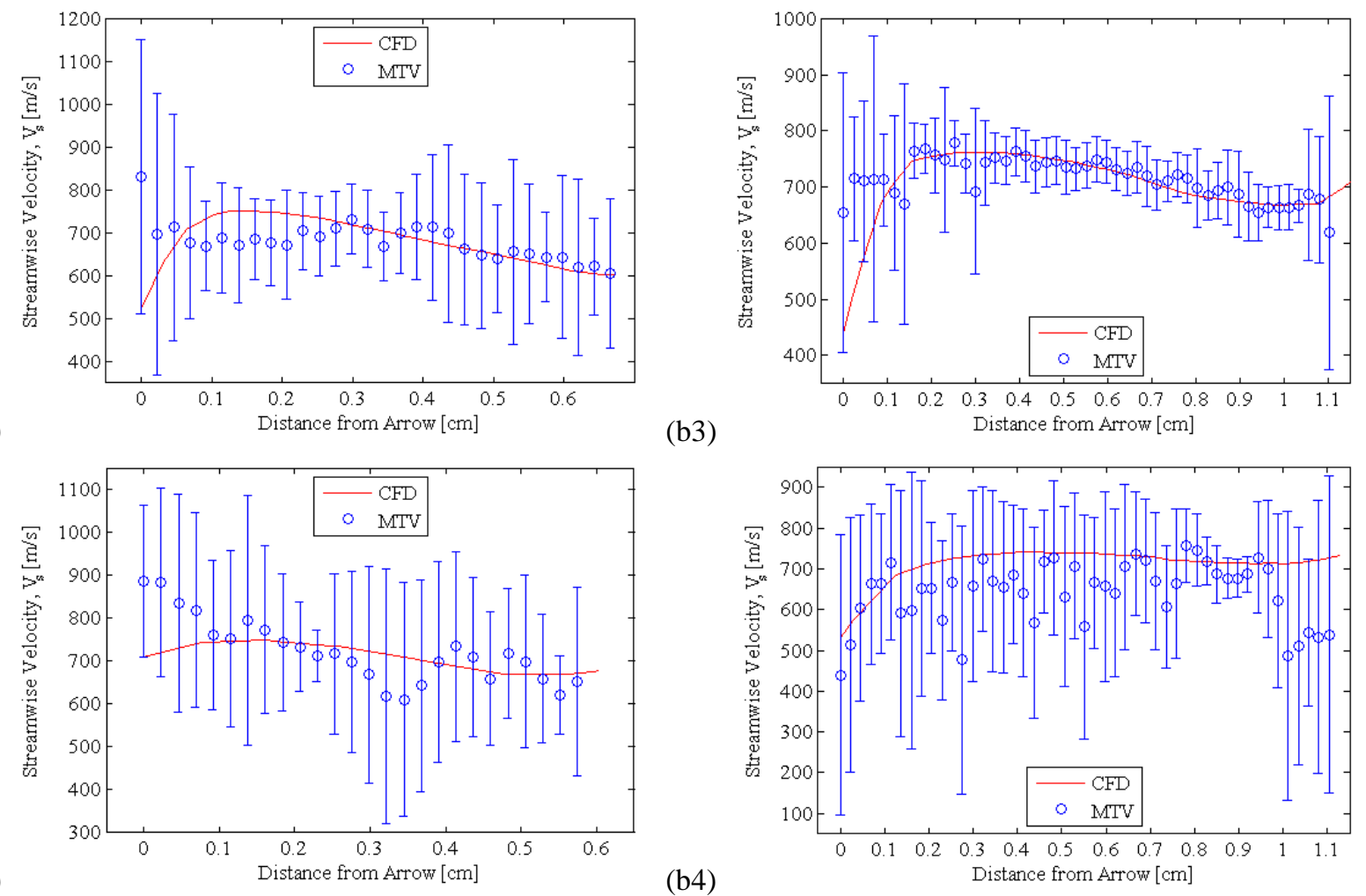

Figure 12. CFD and MTV centerline streamwise velocity comparisons with $P_{0}=350$ psi. Column (a) has $P_{c} \sim 45$ psi. Column (b) has $P_{c} \sim 132$ psi. (a1) and (b1) display the velocities using a color table, where the lines indicate the MTV velocity measurements and the background indicates the CFD velocity measurements. The arrows within (a1) and (b1) indicate which profiles are displayed in (a2)-(a4) and (b2)-(b4), respectively. Within the profiles, the bars extending from the MTV data indicate the measurement uncertainty. 


\section{Discussion and Conclusion}

This work summarizes experimental and computational PLIF flow visualizations of the Orion CEV RCS jets. It compares PLIF MTV velocity measurements with CFD solutions at the same conditions. Furthermore, a more detailed model for CFI PLIF was presented and compared with a simpler model used in past work.

The CFI and PLIF flow visualizations generally agreed in the jet trajectory and shape. The disparities between the theoretical and experimental results can be attributed partly to the CFD solution and partly to the assumptions inherent in the CFI representation of Eq. (1). There could also be inadequate modeling of the NO laser-induced fluorescence process. A likely candidate for the differences between computations and experiment is the assumption that there was no $\mathrm{O}_{2}$ quenching in the flowfield; this could not be avoided in the present work because the CFD solution assumed that air was both the test gas and the RCS jet fluid. Had a unique jet fluid been used in the CFD solution, the model presented herein would be able to account for the effects of $\mathrm{O}_{2}$ quenching. Other possibilities for the discrepancies include saturation of the molecular transitions from high laser intensities, laser absorption by NO, and the time dependence of the fluorescence process, all of which were neglected in the current work. A simplified method of computing the PLIF intensity from CFD, given by $S_{f}$, wilkes was shown to work satisfactorily and is recommended for use in the future. This curve-fitting approach could be extended to include, for example, different concentrations of NO, and possibly $\mathrm{O}_{2}$. Such a model can be distributed to the CFD community to do their own comparisons quickly without knowledge of the underlying spectroscopy. The streamwise velocities of the CFD were mostly within uncertainty of our MTV measurement technique. While not providing a formal validation of the CFD codes, these comparisons give confidence that the main aspects of the RCS jet computation (jet size, shape, trajectory and core velocity) are being adequately modeled.

\section{Acknowledgements}

We wish to acknowledge the contribution to this project from the NASA Langley Research Center 31-Inch Mach 10 Air Tunnel technicians and engineers, including Kevin Hollingsworth, Sheila Wright, Paul Tucker, Tony Robbins, Henry Fitzgerald and Johnny Ellis. This work was supported by the NASA Fundamental Aeronautics Hypersonics Program with support from both the Experimental Capabilities and Aerodynamics-Aerothermodynamics-Plasma Disciplines and the Orion CEV Aeroscience Project (CAP). The authors also wish to thank Greg Buck from NASA Langley Research Center for valuable assistance in performing the experiments, including design, construction and assembly of the model and Craig Johansen, Canadian Natural Sciences and Engineering Research Council Fellow at the National Institute for Aerospace, Hampton VA, for valuable assistance in preparing the manuscript.

\section{References}

\footnotetext{
${ }^{1}$ Ruyten, W. M., Williams, W. D., and Heltsley, F. L., "Computational Flow Imaging for Planar Laser-Induced Fluorescence Applications (CFI PLIF),”AIAA Paper 94-2621, June 1994.

2 Danehy, P. M., Palma, P. C., Boyce, R. R., and Houwing, A. F. P., "Numerical Simulation of Laser-Induced Fluorescence Imaging In Shock-Layer Flows,” AIAA Journal, Vol. 37, No. 6, June 1999, pp. 715-722.

${ }^{3}$ Danehy, P. M., Palma, P. C., Houwing, W. F. P., and McIntyre, T. J., "Comparison of Theoretical Laser-Induced Fluorescence Images with Measurements Performed in a Hypersonic Shock Tunnel," AIAA 1996-2236, 19 $9^{\text {th }}$ AIAA Advanced Measurement and Ground Testing Conference, New Orleans, LA, June 17-20, 1996.

${ }^{4}$ Wilkes (Inman), J. A., Glass, C. E., Danehy, P. M., and Nowak, R. J., "Fluorescence Imaging of Underexpanded Jets and Comparison with CFD," AIAA 2006-0910, 44 $4^{\text {th }}$ AIAA Aerospace Sciences Meeting, January 6-12, 2006.

${ }^{5}$ Hollis, B. R., "Real-Gas Flow Properties for NASA Langley Research Center Aerothermodynamics Facilities Complex Wind Tunnels,” NASA Contractor Report 4755, September 1996.

${ }^{6}$ Micol, J. R., "Langley Aerothermodynamic Facilities Complex: Enhancements and Testing Capabilities," AIAA 19980147, $36^{\text {th }}$ AIAA Aerospace Sciences Meeting, January 12-15, 1998, Reno, Nevada.

${ }^{7}$ G. M. Buck, A. N. Watkins, P. M. Danehy, "Aerothermodynamic Testing of RCS Jet Interactions for the Orion 606D Crew Module in the NASA Langley 31-Inch Mach 10 Air Tunnel: Test 63-CH,” EG-CAP-08-173, April 2009.

${ }^{8}$ Bathel, B. F., Danehy, P. M., Inman, J. A., Jones S. B., Ivey, C. B., and Goyne, C. P., "Multiple Velocity Measurements in Hypersonic Flows Using Sequentially-Imaged Fluorescence Tagging," AIAA 2010-1404, 48 ${ }^{\text {th }}$ AIAA Aerospace Sciences Meeting, January 4-7, 2010, Orlando, Florida.

${ }^{9}$ Danehy, P.M., Alderfer, D.W., Inman, J.A., Berger, K.T., Buck, G.M. and Schwartz, R. J. "Fluorescence Imaging and Streamline Visualization of Hypersonic Flow over Rapid Prototype Wind-Tunnel Models,” Proc. IMechE, Part G: J. Aerospace Engineering, 222(G5), 637-651 (2008).
} 
${ }^{10}$ Danehy, P. M., Inman, J. A., Alderfer, D. W., Buck, G. M., and Bathel, B. F., "Visualization of Flowfield Modification by RCS Jets on a Capsule Entry Vehicle," 2008-1231, 46 ${ }^{\text {th }}$ AIAA Aerospace Sciences Meeting, Reno, Nevada, January 7-10, 2008.

${ }^{11}$ Inman J. A., Danehy, P. M., Alderfer, D. W., and Buck, G. M., and McCrea, A., "Planar Fluorescence Imaging and Three Dimensional Reconstructions of Capsule Reaction-Control-System Jets", AIAA Journal Vol. 47, No. 4, April (2009).

${ }^{12}$ Gnoffo P. A., Gupta R. N., and Shinn J. L., “Conservation Equations and Physical Models for Hypersonic Air Flows in Thermal and Chemical Non-equilibrium”, NASA TP-2867, February 1989.

${ }^{13}$ Dyakonov, A. A., Buck, G. M., DeCaro, A. D., "Analysis of Aeroheating Augmentation due to Reaction Control System Jets on Orion Crew Exploration Vehicle” Paper AIAA 2009-3844, 41st AIAA Thermophysics Conference 22 - 25 June 2009, San Antonio, Texas

${ }^{14}$ Settersten, T. B., Patterson, B. D., and Carter, C. D., "Collisional Quenching of NO $A^{2} \Sigma^{+}\left(v^{\prime}=0\right)$ Between 125 and 294 K,” Journal of Chemical Physics, Vol. 130, 2009.

${ }^{15}$ Eckbreth, A. C., Laser Diagnostics for Combustion Temperature and Species, $2^{\text {nd }}$ ed., Combustion Science \& Technology Book Series, Vol. 3, Taylor \& Francis, New York, New York, 1996.

${ }^{16}$ Di Rosa, M. D., High-Resolution Line Shape Spectroscopy of Transition in the Gamma Bands of Nitric Oxide, Stanford California, Stanford University, 1996.

${ }^{17}$ Paul, P. H., Gray, J. A., Durant Jr., J. L., Thoman Jr., J. W., “Collisional Quenching Corrections for Laser-Induced Fluorescence Measurements of NO $A^{2} \Sigma^{+}$," AIAA Journal, Vol. 32, No. 8, August 1996, pp. 1670-1675.

${ }^{18}$ Ben-Yakar, A., Mungal, G.M., and Hanson, R.K., "Time Evolution and Mixing Characteristics of Hydrogen and Ethylene Transverse Jets in Supersonic Crossflows,” Physics of Fluids, Vol. 18, No. 2, February 2006, Paper 026101.

${ }^{19}$ Gruber, M.R., Nejad, A.S., Chen, T.H., and Dutton, J.C., "Mixing and Penetration Studies of Sonic Jets in a Mach 2 Freestream,” Journal of Propulsion and Power, Vol. 11, No. 2, 1995, pp. 315-323. 